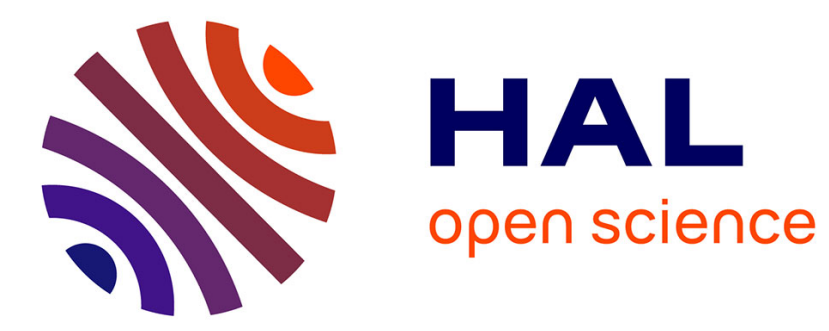

\title{
Théorie des effets de relaxation dans les spectres Mössbauer II. - Méthodes de calcul des formes de raie F. Hartmann-Boutron
}

\section{To cite this version:}

F. Hartmann-Boutron. Théorie des effets de relaxation dans les spectres Mössbauer II. - Méthodes de calcul des formes de raie. Revue de Physique Appliquée, 1983, 18 (7), pp.431-455. 10.1051/rphysap:01983001807043100 . jpa-00245106

\section{HAL Id: jpa-00245106 https://hal.science/jpa-00245106}

Submitted on 1 Jan 1983

HAL is a multi-disciplinary open access archive for the deposit and dissemination of scientific research documents, whether they are published or not. The documents may come from teaching and research institutions in France or abroad, or from public or private research centers.
L'archive ouverte pluridisciplinaire HAL, est destinée au dépôt et à la diffusion de documents scientifiques de niveau recherche, publiés ou non, émanant des établissements d'enseignement et de recherche français ou étrangers, des laboratoires publics ou privés. 


\title{
Théorie des effets de relaxation dans les spectres Mössbauer II. - Méthodes de calcul des formes de raie
}

\author{
F. Hartmann-Boutron \\ Laboratoire de Spectrométrie Physique (*), U.S.M.G., B.P. 68, 38402 St-Martin d'Hères Cedex, France
}

(Reçu le 23 février 1983, accepté le 24 mars 1983)

\begin{abstract}
Résumé. - Cette seconde partie décrit les différentes méthodes (stochastiques et de perturbation) utilisées pour calculer les formes de raie Mössbauer en présence de relaxation, ainsi que leur application à un certain nombre de problèmes concrets.
\end{abstract}

\begin{abstract}
This second part is devoted to a.description of the different methods (stochastic and perturbation) available for computing Mössbauer lineshapes in the presence of relaxation. Their application to some practical problems are indicated.
\end{abstract}

\section{Plan}

1 Introduction.

2 Forme de raie Mössbauer en l'absence de relaxation.

3 Forme de raie en présence de relaxation.

4 Calcul des matrices de relaxation à partir de modèles stochastiques.

5 Calcul des matrices de relaxation par des traitements de perturbation : méthode de la matrice densité et méthode de la résolvante.

6 Traitements de type matrice densité.

7 Traitements de type résolvante.

8 Problèmes propres à la relaxation électronique dipôle-dipôle.

Bibliographie ancienne.

Bibliographie récente, chronologique et commentée.

\section{Introduction.}

Il peut paraître curieux que l'interprétation des spectres Mössbauer en présence de relaxation ait demandé tant d'efforts théoriques, alors que le traitement de la relaxation en R.M.N. (qui relève des mêmes mécanismes physiques) est codifié depuis près de vingt-cinq ans à la suite des travaux, notamment, de Kubo et Tomita, Bloch et Wangness, Redfield, etc. (cf. A. Abragam, Principles of Nuclear Magnetism, chap. VIII).

Ceci tient, entre autres, à deux différences impor-

(*) Laboratoire associé au C.N.R.S. (L.A. 08). tantes entre la résonance magnétique nucléaire et l'effet Mössbauer :

- Les expériences de résonance magnétique nucléaire sont faites en présence d'un champ extérieur appliqué qui joue le rôle de champ quantificateur (la fréquence de Larmor variant de 10 à $200 \mathrm{MHz}$ ) et le signal de résonance ne peut être détecté que si les effets de relaxation sont « petits» $\left(1 / T_{1 \mathrm{n}} \ll 1 \mathrm{MHz}\right.$ dans la plupart des cas).

- Au contraire, pour des raisons diverses, la plupart des expériences Mössbauer sont faites sans champ appliqué et, par ailleurs, les effets de relaxation ne sont observables que s'ils sont au moins comparables à la largeur de raie expérimentale $(\gtrsim 2,2 \mathrm{MHz}$ pour ${ }^{57} \mathrm{Fe}$, $\gtrsim 137 \mathrm{MHz}$ pour ${ }^{170} \mathrm{Yb}$ ) donc relativement importants. Or, le rapport (distance entre raies/ largeur de raie expérimentale) (pouvoir de résolution) n'est pas très grand ( 10 à 100) et, par ailleurs, les taux de relaxation varient très rapidement avec la température, si bien que l'on rencontre fréquemment des situations où l'élargissement dû à la relaxation est comparable à la distance entre raies (spectres " brouillés »). En R.M.N., l'échange chimique peut, lui aussi, conduire à des spectres brouillés, mais la présence du champ quantificateur permet de se ramener à un problème de saut de fréquence (théorie d'Anderson, cf. [2], p. 312). Il n'en est pas de même en Mössbauer, où l'absence de champ quantificateur complique beaucoup les choses.

Dans ce qui va suivre, nous commencerons par rappeler les expressions de la forme de raie Mössbauer 
correspondant aux différents types d'expérience (émission, transmission, diffusion) : nous verrons qu'elles font intervenir les inverses d'une ou de plusieurs supermatrices de Liouville. La relaxation s'introduit dans ces supermatrices sous la forme d'une (super) matrice de relaxation $R$ ou $S$ (cf. première partie [1]).

Le calcul des spectres de relaxation se ramène donc à celui de $R$ ou $S$ qui peut se faire de plusieurs façons :

- soit par des méthodes stochastiques basées sur des modèles phénoménologiques qui permettent de traiter des problèmes très variés, mais ont l'inconvénient de ne pas s'appliquer à très basse température (plus précisément en présence d'orientation nucléaire);

- soit par des méthodes de perturbation (ou $a b$ initio) qui sont valables à toute température mais nécessitent la connaissance du couplage entre l'atome Mössbauer et le bain thermique responsable de sa relaxation. Ces traitements présentent deux variantes :

- méthode de l'équation de mouvement de la matrice densité (dont le principe a été illustré dans [1]),

- méthode de la résolvante.

Nous décrirons le principe et les perfectionnements successifs des différents types de traitements en indiquant quelques applications importantes. Compte tenu de la revue [2] qui couvre la littérature jusqu'en 1975, nous examinerons surtout les résultats acquis depuis cette date. D'autre part, vu la parution récente de la revue de Dattagupta [3] consacrée aux traitements stochastiques, ceux-ci ne seront décrits que brièvement et dans le but de les comparer aux traitements de perturbation. Finalement, le chapitre 8 sera consacré à quelques considérations très élémentaires sur la relaxation dipôle-dipôle.

\section{Forme de raie Mössbauer en l'absence de relaxa- tion.}

Dans ce chapitre, nous calculerons la forme de raie Mössbauer $J(\omega)$ en fonction des superopérateurs d'évolution sans relaxation, $\mathcal{U}_{0}(t)$ et/ou $\mho_{0}(t)$, introduits dans la première partie [1] (Eqs. (80) (75)). Dans le chapitre suivant, nous en déduirons la forme de raie en présence de relaxation, en remplaçant $U_{0}(t)$ et $\mathcal{V}_{0}(t)$ par $\bar{U}(t)$ et $\overline{\mathcal{V}}(t)$ (Eqs. (83) et (78) de [1]).

Nous supposerons que la transition Mössbauer relie un niveau nucléaire excité $I$ et un niveau fondamental $I_{\mathrm{g}}$. Nous supposerons aussi que dans les états $I$ et $I_{\mathrm{g}}$ le noyau est soumis à un hamiltonien $\mathcal{H}_{0}$ (Zeeman, quadrupolaire, hyperfin) sous l'effet duquel $I$ et $I_{\mathrm{g}}$ se décomposent respectivement en sous-niveaux (nucléaires ou électronucléaires) $f$ et $g$.

2.1 FORME DE RAIE D'UNE SOURCE À TEMPÉRATURE ÉLEVÉE. - Lorsque $\mathscr{H}_{\mathrm{o}} / k_{\mathrm{B}} T \ll 1$ (température grande par rapport aux décompositions en sous-niveaux $f$ et $g$ ), on peut démontrer que la forme de raie Mössbauer d'une poudre est donnée par :

$$
\begin{aligned}
J(\omega) \propto \operatorname{Re} \int_{0}^{\infty} \mathrm{d} t \exp [ & \left.\left(i \omega-\frac{\Gamma}{2}\right) t\right] \times \\
& \times \underset{a t}{\operatorname{Trace}}\left\{\underset{\sim}{T}(0) \cdot{\underset{\sim}{T}}_{L}^{\dagger}(t)\right\}
\end{aligned}
$$

ou encore plus explicitement par :

$$
\begin{aligned}
J(\omega) & \propto \operatorname{Re} \int_{0}^{\infty} \mathrm{d} t \exp \left[\left(i \omega-\frac{\Gamma}{2}\right) t\right] \times \\
& \times \sum_{f g} \sum_{M}\left\langle f\left|T_{L}^{M}(0)\right| g\right\rangle\left\langle g\left|T_{L}^{M \dagger}(t)\right| f\right\rangle,
\end{aligned}
$$

où :

- $\omega$ est l'écart en énergie du $\gamma$ par rapport au centre du spectre;

- $\Gamma=1 / \tau_{\mathrm{n}}, \tau_{\mathrm{n}}$ durée de vie de l'état I;

- $T_{L}$, opérateur tensoriel irréductible d'ordre $L$, de composantes $T_{L}^{M}$, est l'opérateur e.l.m.g. qui induit la transition $\gamma(L=1$ pour une transition dipolaire comme dans ${ }^{57} \mathrm{Fe}, \mathrm{M} 1 ; L=2$ pour une transition quadrupolaire comme dans ${ }^{170} \mathrm{Yb}, \mathrm{E} 2$, etc...); les éléments de matrice des $T_{L}^{M}$ par rapport aux états propres $m$ et $m_{g}$ de $I_{z}$ et $I_{z g}$ sont proportionnels à des coefficients de Clebsch-Gordan (théorème de Wigner Eckart) $\left({ }^{1}\right)$,

$$
\text { - } \begin{aligned}
{\underset{\sim}{L}}_{L}(t) & =\exp \left(\frac{i}{\hbar} \mathcal{H}_{0} t\right) T_{L} \exp \left(-\frac{i}{\hbar} \mathcal{H}_{0} t\right) \\
& =U_{0}^{\dagger}(t) \underset{\sim}{T_{L}} U_{0}(t),
\end{aligned}
$$

est le même opérateur en représentation d'Heisenberg en présence du hamiltonien $\mathscr{H}_{0}, U_{0}(t)$ étant l'opérateur d'évolution correspondant à $\mathscr{H}_{0}$ (cf. [1] Eq. (71)).

Comment obtient-on cette expression? Soit par un calcul semi-classique (cf. Schiff, Quantum Mechanics, Sec. 36), soit en calculant la probabilité d'émission spontanée de l'atome sous l'effet de son couplage avec le champ de photons du vide $[4,5]$.

L'équation (1) est analogue à celle qui donne la forme de raie R.M.N. en fonction du signal de précession libre. En effet, ce qu'elle exprime, c'est que pendant la transition Mössbauer il apparaît des éléments off-diagonaux (transitoires) non nuls de l'opérateur $T_{L}$ entre $I$ et $I_{g}: J(\omega)$ est la transformée de Fourier du « signal d'évolution libre " correspondant.

En vue de l'introduction ultérieure de la relaxation, il est intéressant de faire apparaître un superopérateur d'évolution dans l'expression de $J(\omega)$ :

(1) Voir A. R. Edmonds, Angular Momentum in Quantum Mechanics, équations (5.4.1) :

$$
\left\langle\operatorname{Im}\left|T_{L}^{M}\right| I_{g} m_{g}\right\rangle \propto\left\langle L M I_{g} m_{g} \mid L I_{g} I m\right\rangle .
$$

Voir aussi [51] équation (27). 
$J(\omega) \propto \operatorname{Re} \int_{0}^{\infty} \mathrm{d} t \exp \left[\left(i \omega-\frac{\Gamma}{2}\right) t\right] \sum_{f_{1} 1_{M} f_{2} g_{2}}\left\langle f_{1}\left|T_{L}^{M}\right| g_{1}\right\rangle\left\langle g_{1}\left|U_{0}^{\dagger}(t)\right| g_{2}\right\rangle \times$

$$
\times\left\langle g_{2}\left|T_{L}^{M \dagger}\right| f_{2}\right\rangle\left\langle f_{2}\left|U_{0}(t)\right| f_{1}\right\rangle
$$

soit, compte tenu des équations (80) et (83) de [1] :

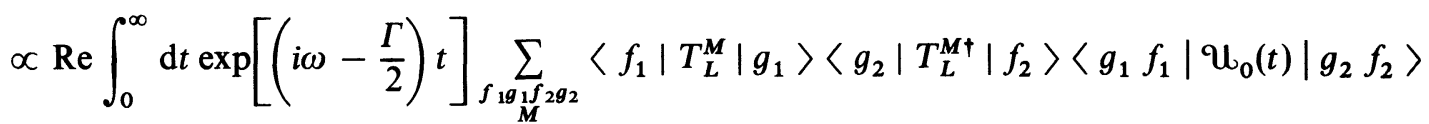

ou encore, compte tenu de l'équation (82) de [1] :

$$
\propto \operatorname{Re} \int_{0}^{\infty} \mathrm{d} t \exp \left[\left(i \omega-\frac{\Gamma}{2}\right) t\right] \sum_{f_{1} g_{1} f_{2} g_{2}}\left\langle f_{1}\left|T_{L}^{M}\right| g_{1}\right\rangle\left\langle g_{2}\left|T_{L}^{M \dagger}\right| f_{2}\right\rangle\left\langle f_{2} g_{2}\left|\vartheta_{0}(t)\right| f_{1} g_{1}\right\rangle .
$$

Si l'on remplace dans $(5) U_{0}(t)$ par $\exp \left(\frac{i}{\hbar} \mathcal{H}_{0}^{\times} t\right)$ et que l'on intègre par rapport au temps, il vient :

$$
\mathfrak{J}(\omega) \propto \operatorname{Re} \sum\left\langle f_{1}\left|T_{L}^{M}\right| g_{1}\right\rangle\left\langle g_{2}\left|T_{L}^{M \dagger}\right| f_{2}\right\rangle\left\langle g_{1} f_{1}\left|\frac{-1}{i \omega-\frac{\Gamma}{2}+\frac{i}{\hbar} \mathscr{H}_{0}^{\times}}\right| g_{2} f_{2}\right\rangle,
$$

donc, pour obtenir la forme de raie, il faut inverser la matrice de Liouville $i \omega-\frac{\Gamma}{2}+\frac{i}{\hbar} \mathfrak{K}_{0}^{\times}$. Si $f, g$ sont états propres de $\mathfrak{H}_{0}$ :

$$
\left\langle g_{1} f_{1}\left|\frac{-1}{i \omega-\frac{\Gamma}{2}+\frac{i}{\hbar} \mathcal{H}_{0}^{\times}}\right| g_{2} f_{2}\right\rangle=\delta_{g_{1 g_{2}}} \delta_{f_{1} f_{2}} \frac{-1}{i \omega-\frac{\Gamma}{2}+\frac{i}{\hbar}\left(E_{g_{1}}-E_{f_{1}}\right)},
$$

et l'on retrouve bien que le spectre se compose de raies de largeur $\Gamma$, de pulsations $\omega=\frac{E_{f}-E_{g}}{\hbar}$ et d'intensités $\left|\left\langle f\left|T_{L}^{M}\right| g\right\rangle\right|^{2}$; ce résultat s'obtiendrait également à partir de l'équation (6).

Nous verrons, dans le chapitre suivant, que l'effet de la relaxation est de remplacer, dans l'équation (7), $\left(i \omega-\frac{\Gamma}{2}+\frac{i}{\hbar} \mathcal{H}_{0}^{\times}\right) \operatorname{par}\left(i \omega-\frac{\Gamma}{2}+\frac{i}{\hbar} \mathcal{H}_{0}^{\times}+R\right)$ où $R$ est une (super) matrice de relaxation.

Notons, pour finir, que la présence dans l'équation (4) du produit $\left\langle g_{1}\left|U_{0}^{\dagger}(t)\right| g_{2}\right\rangle\left\langle f_{2}\left|U_{0}(t)\right| f_{1}\right\rangle$ montre que l'on observe une superposition des évolutions dans l'état fondamental et dans l'état excité. C'est ce qui fait de l'effet Mössbauer une technique moins facile à visualiser que la R.M.N.

2.2 FORME DE RAIE D'UN ABSORBEUR. - Elle est liée à la partie imaginaire de l'indice de réfraction de l'absorbeur [33.1]. A haute température, elle est identique à la forme de raie d'une source (Eqs. (5) et (6) ci-dessus). A basse température, elle est donnée par les équations (34) et (34') de [33.I].

2.3 FoRME DE RAIE D'UNE SOURCE À BASSE TEMPÉRATURE. - Elle s'obtient par un calcul de cascade radioactive ([5] Appendice, [23]).

$J(\omega) \propto \operatorname{Re} \int_{0}^{\infty} \mathrm{d} t^{\prime} \int_{0}^{\infty} \mathrm{d} t^{\prime \prime} \exp \left[\left(i \omega-\frac{\Gamma}{2}\right) t^{\prime}\right] \mathrm{e}^{-\Gamma t^{\prime \prime}} \times \underset{a t}{\operatorname{Trace}}\left\{\underset{\sim}{T_{L}} U_{0}^{\dagger}\left(t^{\prime}\right) \underset{\sim}{T_{L}^{\dagger}} U_{0}\left(t^{\prime}\right) U_{0}\left(t^{\prime \prime}\right) \sigma_{\mathrm{in}}^{I} U_{0}^{\dagger}\left(t^{\prime \prime}\right)\right\}$,

où $\sigma_{\text {in }}^{I}$ est la matrice densité initiale du niveau $I$ au moment où le noyau vient de tomber dans cet état par décroissance radioactive du parent $I_{\mathrm{i}}$.

Cette expression peut se mettre sous la forme plus compacte

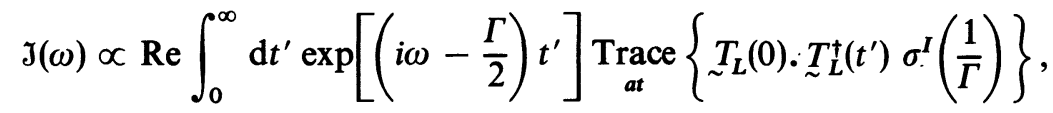


dans laquelle on a posé :

$$
\sigma^{I}\left(\frac{1}{\Gamma}\right)=\Gamma \int_{0}^{\infty} \mathrm{d} t^{\prime \prime} \mathrm{e}^{-\Gamma t^{\prime \prime}} U_{0}\left(t^{\prime \prime}\right) \sigma_{\text {in }} U_{0}^{\dagger}\left(t^{\prime \prime}\right) \mathrm{d} t^{\prime \prime}
$$

$\sigma^{I}(1 / \Gamma)$ peut s'interpréter comme la matrice densité moyenne du niveau $I$ à l'instant d'émission du $\gamma$ Mössbauer (en d'autres termes, pendant le séjour du noyau dans l'état $I$, sa matrice densité $\sigma$ a évolué de $\sigma_{\text {in }}^{I}$ à une valeur moyenne $\sigma^{I}(1 / \Gamma)$, la moyenne portant sur le temps de séjour du noyau dans $I$, qui varie en $\mathrm{e}^{-t / \tau_{\mathbf{n}}}=\mathrm{e}^{-\Gamma t}$ ). A haute température $\left(\mathscr{H}_{0} / k_{\mathrm{B}} T \ll 1\right): \sigma_{\mathrm{in}}^{I}=\sigma^{I}(1 / \Gamma)=1$, et l'on retrouve l'équation (7).

Dans le formalisme de Liouville

$$
\begin{array}{r}
J(\omega) \propto \int_{0}^{\infty} \mathrm{d} t^{\prime} \int_{0}^{\infty} \mathrm{d} t^{\prime \prime} \exp \left[\left(i \omega-\frac{\Gamma}{2}\right) t^{\prime}\right] \mathrm{e}^{-\Gamma t^{\prime \prime}} \times \sum\left\langle f_{1}\left|\underset{\sim}{T_{L}}\right| g_{1}\right\rangle\left\langle g_{2}\left|\underset{\sim}{T_{L}^{\dagger}}\right| f_{2}\right\rangle\left\langle g_{1} f_{3}\left|\mathcal{U}_{0}\left(t^{\prime}\right)\right| g_{2} f_{2}\right\rangle \times \\
\times\left\langle f_{3} f_{1}\left|\vartheta_{0}\left(t^{\prime \prime}\right)\right| f_{4} f_{5}\right\rangle\left\langle f_{4}\left|\sigma_{\text {in }}^{I}\right| f_{5}\right\rangle,
\end{array}
$$

que l'on peut transformer comme l'équation (5).

2.4 FoRME DE RAIE EN DIFFUSION. - Son calcul est très long et très compliqué, cf. [33.I] : $J=I_{1}+I_{2}+I_{3}$ où $I_{1}, I_{2}, I_{3}$ sont donnés par les équations (45)-(47) ou (48)-(50) de [33.I].

\section{Forme de raie en présence de relaxation.}

Elle s'obtient (cf. première partie [1]) en remplaçant dans les expressions obtenues en l'absence de relaxation $\mathcal{U}_{0}(t)$ par $\bar{u}(t)=\exp \left[\left(+\frac{i}{\hbar} \mathcal{H}_{0}^{\times}+R\right) t\right]$, ou $\mathcal{v}_{0}(t)$ par $\bar{v}(t)=\exp \left[\left(-\frac{i}{\hbar} \mathcal{H}_{0}^{\times}+S\right) t\right]$ (où $R$ et $S$ sont des supermatrices de relaxation) et en effectuant les intégrations par rapport au temps. Signalons que lorsque la relaxation intervient plus d'une fois dans le problème (cas de la source à basse température et de la diffusion) la justification de cette règle n'est pas triviale (cf. [23] Appendice I).

3.1 SOURCE OU ABSORBEUR À HAUTE TEMPÉRATURe ([6], [23] EQ. (45)).

$$
J(\omega) \propto \operatorname{Re} \sum_{\substack{f_{1} f_{2} \\ g_{1} g_{2}}}\left\langle f_{1}\left|\underset{\sim}{T_{L}}\right| g_{1}\right\rangle\left\langle g_{2}\left|\underset{\sim}{T_{L}^{\dagger}}\right| f_{2}\right\rangle\left\langle g_{1} f_{1}\left|\frac{-1}{i \omega-\Gamma / 2+(i / \hbar) \mathcal{H}_{0}^{\times}+R}\right| g_{2} f_{2}\right\rangle
$$

qui peut d'ailleurs également s'écrire (cf. [1] Eq. (82)) :

$$
J(\omega) \propto \operatorname{Re} \sum_{\substack{f_{1} f_{2} \\ g_{1} g_{2}}}\left\langle f_{1}\left|\underset{\sim}{T_{L}}\right| g_{1}\right\rangle\left\langle g_{2}|\underset{\sim}{T}| f_{2}^{\dagger}\right\rangle\left\langle f_{2} g_{2}\left|\frac{-1}{i \omega-\Gamma / 2-(i / \hbar) H_{0}^{\times}+S}\right| f_{1} g_{1}\right\rangle
$$

$J(\omega)$ fait intervenir une matrice de Liouville.

3.2 AbSORBeUr En ÉQUilibre thermiQue À baSSE TEMPÉratURe. - (Cf. [33. II] Eq. (5)) :

$$
J(\omega) \propto \operatorname{Re} \sum_{\substack{f_{1} f_{2} \\ g_{1} g_{2}}} \sigma_{g_{11} g_{1}}\left\langle f_{1}\left|\underset{\sim}{T_{L}}\right| g_{1}\right\rangle\left\langle g_{2}\left|\underset{\sim}{T_{L}^{\dagger}}\right| f_{2}\right\rangle\left\langle g_{1} f_{1}\left|\frac{-1}{i \omega-\Gamma / 2+(i / \hbar) \mathcal{H}_{0}^{\times}+R}\right| g_{2} f_{2}\right\rangle .
$$

Dans cette équation $\sigma_{g_{1 g_{1}}}$ est un élément de la matrice densité de Boltzmann du niveau $I_{g}(\sigma=0$ à haute température). $J(\omega)$ ne contient qu'une matrice de Liouville.

3.3 SOURCE À baSSE TEMPÉRATURe ([7], [23] EQ. (44)).

$$
J(\omega) \propto \operatorname{Re} \sum_{\substack{f_{1} f_{2} f_{3} \\ g_{12}}}\left\langle f_{1}\left|{\underset{\sim}{L}}_{L}\right| g_{1}\right\rangle\left\langle g_{2}\left|\underset{\sim}{T_{L}^{\dagger}}\right| f_{2}\right\rangle \times\left\langle g_{1} f_{3}\left|\frac{-1}{i \omega-\frac{\Gamma}{2}+\frac{i}{\hbar} \mathcal{H}_{0}^{\times}+R}\right| g_{2} f_{2}\right\rangle \sigma_{f_{3} f_{1}}^{I}\left(\frac{1}{\Gamma}\right),
$$


avec

$$
\sigma_{f_{3} f_{1}}^{I}\left(\frac{1}{\Gamma}\right)=\sum_{f_{4} f_{5}}\left\langle f_{3} f_{1}\left|\frac{-1}{-\Gamma-\frac{i}{\hbar} \mathcal{H}_{0}^{\times}+S}\right| f_{4} f_{5}\right\rangle\left\langle f_{4}\left|\sigma_{\text {in }}^{I}\right| f_{5}\right\rangle .
$$

$\mathfrak{J}(\omega)$ fait intervenir deux matrices de Liouville.

On remarque que dans les équations (13) (13") (14) la supermatrice $\left(i \omega-\Gamma / 2+(i / \hbar) J_{0}^{x}+R\right)^{-1}$, qui dépend de la fréquence, contrôle le profil de raie, tandis que dans $\left(13^{\prime \prime}\right)$ et $(14) \sigma_{g_{1} g_{1}}$ et $\sigma_{f_{3} f_{1}}^{I}(1 / \Gamma)$ contrôlent l'intensité des raies.

L'interprétation physique de (15) est la suivante : dans une source en présence de relaxation, pendant le séjour du noyau dans l'état transitoire $I$, sa matrice densité tend à évoluer de la valeur initiale $\sigma_{\text {in }}^{I}$ (due au peuplement par le parent) vers la valeur de Boltzmann $\sigma_{\mathrm{B}}^{I}$, mais cette évolution est interrompue au stade intermédiaire $\sigma^{I}(1 / \Gamma)$ par la décroissance radiative de $I$. Dans la base des états propres de $\mathscr{H}_{0}$ la matrice densité du parent est diagonale (puisqu'il est à l'équilibre thermique) et il en est de même pour $\sigma_{\text {in }}^{I}$, puisque $I$ est peuplé par émission spontanée; donc $f_{4}=f_{5}$ dans l'équation (15). Par ailleurs, dans tous les cas étudiés, $\sigma^{I}$ reste diagonale au cours de la relaxation. donc $f_{3}=f_{1}$ dans (14) et dans (15) qui devient :

$$
\begin{array}{r}
\sigma_{f_{1} f_{1}}^{I}\left(\frac{1}{\Gamma}\right)=\sum_{f_{4}}\left\langle f_{1} f_{1}\left|\frac{-1}{-\Gamma+S}\right| f_{4} f_{4}\right\rangle \times \\
\times\left\langle f_{4}\left|\sigma_{\text {in }}^{I}\right| f_{4}\right\rangle .
\end{array}
$$

L'équation $\left(15^{\prime}\right)$ est une relation entre populations initiales et finales des niveaux

$$
p_{\text {in } f_{4}}^{I}=\left\langle f_{4}\left|\sigma_{\text {in }}^{I}\right| f_{4}\right\rangle, \quad p_{f_{1}}^{I}\left(\frac{1}{\Gamma}\right)=\sigma_{f_{1} f_{1}}^{I}\left(\frac{1}{\Gamma}\right) .
$$

C'est la raison pour laquelle on parle de « réorientation des populations » (la disparition de $\mathcal{H}_{0}^{\times}$entre (15) et $\left(15^{\prime}\right)$ vient dę ce que les populations commutent avec $\left.\varkappa_{0}\right)$. En pratique, les populations moyennes $p_{f}(1 / \Gamma)$ peuvent être obtenues sans inverser de matrice de Liouville, en écrivant des équations de bilan détaillé du type ([5] p. 294) :

$$
\begin{aligned}
0=\frac{\mathrm{d} p_{f}}{\mathrm{~d} t}=\Gamma_{i} p_{f}^{\text {in }}-\Gamma p_{f}-\sum_{f^{\prime}} & W_{f \rightarrow f^{\prime}} p_{f}+ \\
& +\sum_{f^{\prime}} W_{f^{\prime} \rightarrow f} p_{f^{\prime}}
\end{aligned}
$$

dans lesquelles les termes en $\Gamma_{i}$ et $\Gamma$ représentent l'alimentation et la désexcitation radiatives de $I$ et les termes en $W$ correspondent à la relaxation (cf. [8] Eq. (28)).

Pour que les effets de réorientation soient importants il faut, d'une part, que $\sigma_{\text {in }}^{I}, \sigma^{I}(1 / \Gamma) \neq 1$, ce qui exige des très basses températures $\left(T \lesssim \mathscr{H}_{0}^{\mathrm{x}} / k_{\mathrm{B}} \sim\right.$ $10^{-2}-10^{-1} \mathrm{~K}$ ), d'autre part, que les taux de relaxation $W$ soient comparables à $\Gamma=1 / \tau_{\mathrm{n}}$ (c'est-à-dire $S \sim$
$R \sim \Gamma$ ). Dans ce cas, le spectre est résolu, avec des raies légèrement élargies (puisque $R$ intervient aussi dans la matrice de Liouville dépendant de $\omega$ ) et présentant des anomalies d'intensité liées aux $p_{f}(1 / \Gamma)$. Quand la température croît $\left(T \gg H_{0}^{x} / k_{\mathrm{B}}\right), \sigma_{\text {in }}^{I}$ et $\sigma^{I}(1 / \Gamma)$ deviennent égales à 1 , mais $R$ croît également et peut devenir comparable à $\mathcal{H}_{0}^{x}$. C'est alors la première matrice de Liouville (dépendant de la fréquence) qui commande l'aspect du spectre : on observera un spectre brouillé pour $R \lesssim H_{0}^{x} / \hbar$ et une raie unique pour $R \gg H_{0}^{x} / \hbar$. Les sources fournissent donc deux fenêtres d'étude de la relaxation : $R \sim \Gamma$ et $R \sim$ $\mathcal{H}_{0}^{\mathrm{x}} / \hbar[7,8]$. Au contraire, l'absorbeur ne permet d'atteindre que la fenêtre $R \sim \mathscr{H}_{0}^{x} / \hbar$.

3. 4 Diffusion. - Cf. [33.II] equation (9) : J( $\omega)$ est la somme de 3 produits de 3 matrices de Liouville. Comme les expériences de source, la diffusion donne accès aux deux fenêtres : $S \sim \mathcal{H}_{0}^{\mathrm{x}} / \boldsymbol{h}$ et $S \sim \Gamma$ (sans la restriction $k_{\mathrm{B}} T \lesssim H_{0}^{x}$ dans ce dernier cas). Mais les formules sont tellement lourdes que seule la fenêtre $S \sim \Gamma$ paraît exploitable de façon simple (cf. [33.II] p. 552-555: la relaxation se traduirait par l'apparition de raies nouvelles dans le spectre diffusé).

3.5 Remarques. - Dans les équations (13), (13') et (14), l'expression de la matrice de Liouville qui dépend de la fréquence est relative à un rayonnement monochromatique. Pour tenir compte de la largeur $\Gamma^{\prime}$ de l'absorbeur (dans une expérience d'émission) ou de la source (dans une expérience de transmission), il faudrait remplacer $\frac{\Gamma}{2} \operatorname{par} \frac{\Gamma+\Gamma^{\prime}}{2}$ (par contre (15) restera inchangée). Les équations (5), (6) et (9) de [33.II] tiennent compte de $\Gamma^{\prime}$.

- Dans l'équation $\left(15^{\prime}\right)$ les valeurs propres de la matrice de relaxation $S$ sont négatives : $(-\Gamma+S)$ ne s'annule jamais. Il aurait peut-être mieux valu adopter une autre convention de signe pour la matrice de relaxation...

\section{Calcul des matrices de relaxation à partir de modèles stochastiques.}

Ces modèles se divisent en deux grandes catégories :

- Dans la première, l'atome est soumis à un hamiltonien aléatoire qui saute entre plusieurs valeurs, commutant ou non entre elles.

- Dans la deuxième, l'atome subit des chocs aléatoires distribués au hasard dans le temps. 
4.1 Hamiltonien aléatoire SAUtant entre pluSIEURS VAlEURS. - Dans ce type de traitement le hamiltonien de relaxation est constitué par des " champs externes » (suivant l'expression de Clauser et Blume [9]) aléatoires (champ magnétique ou gradient de champ électrique) agissant directement sur le noyau. Par exemple :

$$
\mathscr{H}_{1}=-\hbar\left\{\begin{array}{l}
\gamma_{\mathrm{n}}^{I} \mathbf{I} \cdot \mathbf{H}(t) \\
\gamma_{\mathrm{n}}^{I_{g}} \mathbf{I}_{g} \cdot \mathbf{H}(t)
\end{array}\right.
$$

Le cas le plus simple est constitué par un champ fluctuant qui saute aléatoirement entre les 2 valeurs $H_{\mathrm{z}}= \pm H$ avec une pulsation de saut $\Omega=1 / \tau$. Ce problème peut être traité par une méthode due à P. W. Anderson : cf. [2] p. 312. Rappelons que si $\Omega$ est petit par rapport à $\omega_{\mathrm{n}}^{I, I_{g}}=-\gamma_{\mathrm{n}}^{I, I_{g}} H$, on observe un spectre Zeeman unique qui est la superposition de deux spectres identiques associés à $\pm H$. Si $\Omega$ croît, les raies s'élargissent et le spectre se brouille pour $\Omega \sim \omega_{\mathrm{n}}^{I, I_{g}}$. Enfin, quand $\Omega$ devient très grand, le spectre se réduit à une seule raie (" rétrécissement extrême ").

Ce modèle peut être adapté au cas d'un champ qui prend plusieurs valeurs, $H_{1}, H_{2}, H_{3} \ldots$ inégales en module et en probabilité. Il apparaît alors qu'en relaxation lente, le spectre est la surperposition de plusieurs spectres Zeeman correspondant à $H_{1}, H_{2}$, $\mathrm{H}_{3}$, tandis qu'en relaxation rapide, il se réduit à un spectre unique correspondant à $\langle H\rangle$. Il en est ainsi dans un corps magnétique ordonné, où l'on peut considérer que le moment électronique $g \mu_{\mathrm{B}} \mathrm{S}$ qui crée $\mathbf{H}$ saute rapidement entre ses niveaux dans le champ moléculaire (ou bien encore que I ne suit pas les modulations rapides de $\mathbf{S}$ par les ondes de spin), de sorte que le champ hyperfin est proportionnel à l'aimantation.

L'observation d'un spectre Zeeman non élargi signifie donc :

a) soit que le champ saute lentement entre plusieurs vàleurs égales en module (par ex. $H_{\mathrm{z}}= \pm H$ ),

b) soit qu'il saute rapidement entre plusieurs valeurs inégales en module, si bien que le noyau voit un " champ effectif » $\langle\boldsymbol{H}\rangle$.

Le fait qu'un spectre Zeeman bien résolu puisse correspondre aux deux situations très différentes $a$ ) et $b$ ) devrait être davantage pris en compte dans l'étude de la transition verre de spin.

Le modèle d'Anderson permet également de décrire la relaxation paramagnétique par un couplage anisotrope $\mathscr{H C}_{1}=A_{\|} I_{\mathrm{z}} S_{\mathrm{z}}(t)$ (en particulier dans les doublets de Kramers des terres rares), le superparamagnétisme, etc... Toutes ces applications sont discutées en détail dans la référence [2] p. 313-315.

Jusqu'à présent, nous avons supposé que $\mathscr{H}_{1}(t)$ sautait entre plusieurs valeurs commutant entre elles. Le cas où $\mathcal{H}_{1}(t)$ saute entre $n$ valeurs quí ne commutent pas entre elles a été abordé par Blume et Tjon (1968) sur des exemples particuliers [10] $\left({ }^{2}\right)$ puis par Blume (1968) sous forme générale [11]. Blume introduit le formalisme de Liouville et montre que $J(\omega)$ s'obtient par inversion d'une matrice $N \times N$ avec $N=$ $\cdot(2 I+1)\left(2 I_{g}+1\right) \times n\left({ }^{3}\right)$. Plus précisément (cf. [11] Eqs. (21), (22)) la forme de raie Mössbauer haute température est directement reliée à la transformée de Laplace $\bar{\Psi}(p)\left(\operatorname{avec} p=\frac{\Gamma}{2}-i \omega\right)$ du superopérateur d'évolution de l'atome, moyennée sur les états stochastiques $a, b$ correspondant aux différentes valeurs $V_{j}^{\times}$ que peut prendre $\mathfrak{H}_{1}^{\times}(t)$ :

$$
\mathfrak{J}(\omega) \sim \overline{\mathcal{U}}(p)=\sum_{a b} p_{a}\langle a|\mathcal{U}(p)| b\rangle,
$$

avec :

$$
\mathcal{U}(p)=\frac{1}{p \underset{\approx}{1}-W-i \sum_{j} V_{j}^{\times} F_{j}},
$$

où la matrice $W$ décrit les probabilités de sauts entre les états stochastiques $a, b$, tandis que $p_{a}, p_{b}$ sont les probabilités d'occupation de ces états et $F_{j}$ est une matrice stochastique définie $\operatorname{par}\left\langle a\left|F_{j}\right| b\right\rangle=\delta_{a b} \delta_{a j}$.

La dimension $N$ de la matrice $\mathcal{U}(p)$ a été ramenée à $(2 I+1)\left(2 I_{g}+1\right)$ par Dattagupta et Blume (1974) [12] moyennant l'utilisation d'un modèle de saut particulier baptisé R.P.A. (Random Phase Approximation) qui consiste à dire que le système n'a pas de mémoire. Dans ce cas $W_{a b}=\lambda p_{b}$ où $\lambda$ est une fréquence caractéristique de saut. On démontre alors (cf. [12] Eqs. (13) et (14)), que :

$$
\overline{u_{u}}(p)=\bar{u}_{0}(p+\lambda) /\left[1-\lambda \bar{u}_{0}(p+\lambda)\right]
$$

où $\bar{\Psi}_{0}(p)$ correspond au spectre en l'absence de sauts :

$$
\overline{\mathcal{U}}_{0}(p)=\sum_{j} p_{j}\left[p \underset{\approx}{1}-i V_{j}^{\times}\right]^{-1} .
$$

Ce modèle a été appliqué au cas isotrope où le « champ externe " fluctuant peut prendre n'importe quelle direction dans l'espace avec une égale probabilité. Pour ceci on remarque d'abord (cf. [12] Eqs. (32), (33), [30] Eq. (27)), que la forme de raie Mössbauer d'une poudre peut s'écrire :

$$
J(\omega) \propto \sum_{M} G_{L L}^{M M}\left(p=\frac{\Gamma}{2}-i \omega\right),
$$

où les $G_{L L}^{M M}(p)$ sont les transformées de Laplace de "facteurs de perturbation" $G_{L L}^{M M}(t)$ analogues à

$\left({ }^{2}\right)$ Le modèle de Blume et Tjon a été adapté par Bonville et al. (1981) [61] au calcul des spectres de relaxation de $\mathrm{Fe}^{++}$ dans $\mathrm{ZnS}$ cubique, où l'ion $\mathrm{Fe}^{++}$saute entre des niveaux de spin-orbite $\Gamma_{4}$ (triplet) et $\Gamma_{1}$ (singulet), ainsi qu'à l'intérieur du triplet $\Gamma_{4}$.

$\left({ }^{3}\right)$ Pour ${ }^{57} \mathrm{Fe}$ et un champ sautant entre 2 valeurs $H_{z}=$ $\pm H$ la matrice est $16 \times 16$. Avec le traitement de type Anderson elle se factorise en 8 matrices $2 \times 2$, identiques deux à deux; les règles de sélection font qu'on n'en utilise que 6. 
ceux utilisés en corrélations angulaires perturbées. Autrement dit, si l'on fait un développement multipolaire de la partie off-diagonale ${ }^{I, I_{g}} \sigma$ de la matrice densité de l'atome Mössbauer suivant des opérateurs tensoriels irréductibles normés $\left({ }^{4}\right)\left({ }^{I, I_{g}} T_{L}\right)$ :

$$
{ }^{I, I_{g}} \sigma=\sum_{L^{\prime} M^{\prime}}\left({ }^{I, I_{g}} \sigma_{L^{\prime}}^{M^{\prime} *}\right)\left({ }^{I, I_{g}} T_{L^{\prime}}^{\left.M^{\prime}\right)} \quad\left(\left|I-I_{g}\right| \leqslant L^{\prime} \leqslant I+I_{g}\right),\right.
$$

les $G_{L^{\prime \prime} L^{\prime}}^{M^{\prime \prime} M^{\prime}}(t)$ décrivent les transformations des composantes multipolaires $\left({ }^{I, I_{g}} \sigma_{L}^{M *}\right)$ les unes dans les autres :

$$
\left({ }^{I, I_{g}} \sigma_{L^{\prime}}^{M^{\prime} *}(t)\right)=\sum_{L^{\prime \prime} M^{\prime \prime}} G_{L^{\prime \prime} L^{\prime}}^{M^{\prime \prime} M^{\prime}}(t)\left({ }^{I, I_{g}} \sigma_{L^{\prime \prime}}^{M^{\prime \prime}} *(0)\right)
$$

$$
G_{L L}^{0}(p+\lambda)=\sum_{m_{I}, m_{I_{g}}}\left(\begin{array}{cc}
I & I_{g} \\
m_{I} & -m_{I_{g}}
\end{array}\right.
$$

Ces formules permettent d'obtenir aisément des expressions analytiques pour $\mathfrak{J}(\omega)$ (Ex. : fluctuations du G.C.E. dans un liquide, cf. [12] Eq. (44); champ hyperfin se réorientant aléatoirement dans tout l'espace cf. [62] Eq. (4.36)).

L'équation (13) de Dattagupta et Blume [12] (cf. Eq. (19) ci-dessus) a été adaptée par Sivardière et Blume (1975) [25] au cas de la relaxation paramagnétique $\left(\mathscr{H}_{0}=\mathbf{I} \cdot \boldsymbol{A} . \mathbf{S}\right)$, en présence de "chocs " qui désorientent cỗmplètement le spin électronique $\mathbf{S}$ (cf. Eq. (31) ci-dessous); malheureusement, dans leurs équations (3) à (11), ces auteurs emploient la notation $G$ au lieu de $U$. Le principe de leur calcul est le suivant :

- Recherche des $(2 I+1)\left(2 I_{g}+1\right)(2 S+1)^{2}$ états propres de $\mathcal{H}_{0}^{\mathrm{x}}$.

- Calcul de $G_{0}(p+\lambda) \equiv\left(p+\lambda-i \mathcal{H}_{0}^{\times}\right)^{-1}$ dans cette base.

- Passage à la base découplée $\left|M_{I} M_{s}, M_{I_{g}}^{\prime} M_{s}^{\prime}\right\rangle$.

- Moyenne sur les états électroniques $M_{s}, M_{s}^{\prime}$ ce qui donne $\bar{G}_{0}(p+\lambda)$ (Eq. (5) de [25]) (matrice de dimension $\left.(2 I+1)\left(2 I_{g}+1\right)\right)$.

- Calcul de $\bar{G}(p)$, équation (11) de [25].

En pratique, cette méthode complexe n'est véritablement utile que dans le cas anisotrope (par exemple quadruplet $\Gamma_{8}$ [25]). En effet, dans le cas isotrope (Ex. : Fig. 1 de [25]) le spectre peut être obtenu beaucoup plus simplement par adaptation du modèle de Scherer-Blume (cf. ci-dessous).

4.2 ModÈle DE CHOCS ALÉATOIRES DISTRIBUÉS AU HASARD DANS LE TEMPS. - La première théorie de ce genre a été élaborée par Clauser et Blume (1971) [9] pour traiter la relaxation paramagnétique dans le cas isotrope où le hamiltonien $\mathcal{H}_{1}=A \mathbf{I} . \mathbf{S}(t)$ prend un continuum de valeurs qui ne commutent pas entre elles. Mais au lieu de faire sauter le hamiltonien entre ces valeurs, comme dans le paragraphe précédent,

$\left({ }^{4}\right)$ Cf. equation (27) de [51].
L'équation (21) traduit alors le fait que l'expérience Mössbauer n'est sensible qu'à l'évolution de la composante multipolaire d'ordre $L$ correspondant à l'opéra$\operatorname{teur}^{I, I_{g}} T_{L}$ qui induit la transition $\gamma$.

Ceci dit, lorsque la relaxation est isotrope on peut démontrer ([12] Eqs. (39) (40)) que :

$$
G_{L L}^{M M}(p) \equiv G_{L L}(p)=\frac{G_{L L}^{0}(p+\lambda)}{1-\lambda G_{L L}^{0}(p+\lambda)},
$$

où $G_{L L}^{0}$ est le facteur de perturbation en l'absence de sauts, dans un système d'axes où le hamiltonien fluctuant instantané $V$ est diagonal $\left({ }^{5}\right)$ :

$$
\left.\begin{array}{c}
L \\
M
\end{array}\right)^{2} /\left[p+\lambda+i\left(V_{m_{I}}^{I}-V_{m_{I_{g}}}^{I_{g}}\right)\right]
$$

Clauser et Blume supposent (assez artifíciellement) que l'atome est soumis en permanence à un hamiltonien statique $\mathscr{H}_{0}=A \mathbf{I} . \mathbf{S}$ et que de temps en temps $\mathbf{S}$ subit un choc $V_{i} \delta\left(t-t_{i}\right)$ qui le désoriente.

Sous forme générale (cf. [9], et [2] p. 316), en présence de $H_{0}$ et de chocs $V_{i} \delta\left(t-t_{i}\right)$, l'opérateur d'évolution $U$ du système s'écrit :

$$
\begin{array}{r}
U(t)=U_{0}\left(t-t_{i}\right) T_{i} U_{0}\left(t_{i}-t_{i-1}\right) \ldots U_{0}\left(t_{2}-t_{1}\right) \times \\
\times T_{1} U_{0}\left(t_{1}\right),
\end{array}
$$

où $U_{0}$ représente l'évolution sous l'effet du seul $\mathscr{H}_{0}$ et où $T_{i}=\exp \left(-\frac{i}{\hbar} V_{i}\right)$. Clauser et Blume passent en représentation de Liouville :

$$
\begin{aligned}
\mathcal{u}_{0}(t) & =\exp \left(\frac{i}{\hbar} \mathcal{H}_{0}^{\times} t\right) \\
\nearrow & =\exp \left(\frac{i}{\hbar} V^{\times}\right),
\end{aligned}
$$

et font l'hypothèse que les chocs sont répartis dans le temps suivant une loi de Poisson ([2] Eqs. (VI.41-42)). Ils trouvent alors que la transformée de Laplace $\bar{\Psi}(p)$ de l'opérateur d'évolution est donnée (après avoir moyenné sur l'opérateur de choc : $\mathfrak{C} \rightarrow \mathcal{C}_{a v}$ ) par une progression géométrique de somme :

$$
\begin{aligned}
\bar{U}(p) & =\frac{\mathcal{U}_{0}(p+\lambda)}{1-\lambda \mathcal{G}_{a v} \mathcal{U}_{0}(p+\lambda)} \\
& =\frac{1}{p-\frac{i}{\hbar} \mathscr{H}_{0}^{\times}-\lambda\left(\mathscr{C}_{a v}-1\right)},
\end{aligned}
$$

(5) La quantité $\left(\begin{array}{ccc}I & I_{g} & L \\ m_{I} & -m_{I_{g}} & M\end{array}\right)$ dans l'équation (25) est un symbole « $3 \mathrm{j}$ 》 de Wigner, qui est une forme symétrisée du coefficient de Clebsch-Gordan, cf. Edmonds, Angular Momentum in Quantum Mechanics equation (3.7.3). 
$\lambda$ caractérise la distribution de Poisson des chocs (la probabilité pour qu'il y ait $n$ chocs à $t_{1} \ldots t_{n}$ entre 0 et $t$ est $\left.\lambda^{n} \mathrm{e}^{-\lambda t}\right)$.

Clauser et Blume ont considéré le cas d'un atome qui a une structure hyperfine $\mathscr{H}_{0}=A \mathrm{I} . \mathrm{S}$ dans l'état excité, $\mathscr{H}_{0}=A_{g} \mathbf{I}_{g}$. S dans l'état fondamental, et dont le spin $\mathbf{S}$ est soumis à des impulsions de champ : $V_{i}=H_{i} \cdot \mathbf{S}$. Dans ce cas la matrice de Liouville à inverser a pour dimension $N=(2 I+1)\left(2 I_{g}+1\right)$ $(2 S+1)^{2}$. Le calcul de la forme de raie par cette méthode a été effectué par Gonzalez et al. [6] pour $\mathrm{AuYb}^{170}\left(I=2, I_{g}=0, S=1 / 2\right.$, d'où $\left.N=20\right)$ et leur a servi de guide pour l'utilisation de la théorie de perturbation de Hirst (voir ci-dessous).

Les résultats de Clauser-Blume ont également été appliqués par Sivardière-Blume-Clauser (1975) [24] au calcul des spectres de relaxation de ${ }^{57} \mathrm{Fe},{ }^{166} \mathrm{Er}$, ${ }^{169} \mathrm{Tm}$ associés à des doublets électroniques possédant une structure hyperfine anisotrope ou isotrope [24].

En fait, pour une structure hyperfine isotrope et un spin $S=1 / 2$, il est possible d'arriver plus rapidement au même résultat par adaptation au cas Mössbauer (Chopin et al. (1976) [30]) d'un modèle élaboré par Scherer [13] et Blume [14] (1971) pour les corrélations angulaires perturbées. Ce modèle postule des chocs de type collision forte désorientant complètement $\mathbf{S}$ ([2] p. 337-339) :

$$
\left\langle m_{s} m_{s}^{\prime}\left|\mathscr{C}_{a v}\right| n_{s} n_{s}^{\prime}\right\rangle=\frac{1}{2 S+1} \delta_{m_{s} m^{\prime} s} \delta_{n_{s} n^{\prime}} .
$$

Il permet d'établir une relation analogue à (29), mais pour le facteur de perturbation (cf. Eq. (24)) :

$$
G_{L L}(p) \equiv G_{L L}^{M M}(p)=\frac{G_{L L}^{0}(p+\lambda)}{1-\lambda G_{L L}^{0}(p+\lambda)}
$$

La forme de raie de $A u Y^{170}$ peut ainsi être obtenue sans aucune diagonalisation (Chopin et al. [30] Eqs. (26)-(34); [2] p. 339 Eq. (VII-101); Eq. (48) ciaprès). Plus généralement pour $S=1 / 2$ et une symétrie sphérique les trois théories : Clauser-Blume [9], Sivardière-Blume [25], Chopin et al. [30] sont équivalentes, cette dernière étant de loin la plus facile à mettre en œuvre.

Par contre, pour $S \neq 1 / 2$, le modèle de SchererBlume et Sivardière-Blume (Eq.(31)) ne donne pas une description réaliste de la relaxation électronique (cf. discussion dans [2] p. 341), tandis que celui de ClauserBlume (relaxation par un champ fluctuant) garde un sens physique.

4.3 HAMILTONIEN SAUTANT ENTRE PLUSIEURS VALEURS SOUS L'EFFET DE CHOCS ALÉATOIRES. - Aussi bien dans la théorie de Clauser-Blume que dans celle de type Scherer-Blume, le modèle de relaxation utilisé est de type "spectre blanc", c'est-à-dire que la matrice de relaxation ne dépend pas des fréquences hyperfines associées à $\mathcal{H}_{0}^{\times}$.

Cette dernière limitation a été levée par Dattagupta (1977). Pour ceci, Dattagupta postule [40] que l'atome est soumis entre les chocs à un hamiltonien pouvant prendre $n$ valeurs :

$$
\mathscr{H}_{0}+V_{a}, \mathscr{H}_{0}+V_{b}, \ldots
$$

et qu'il saute entre ces valeurs sous l'effet $\mathscr{C}$ des chocs. Le superopérateur d'évolution entre 2 états stochastiques $a$ et $b$ prend donc la forme ([40] Eq. (9))

$$
\begin{array}{r}
(a|\mathcal{U}(t)| b)=\sum_{c d e} \mathrm{e}^{i\left(\mathcal{X e}_{0}^{\times}+V_{a}^{\times}\right) t_{1}} \mathcal{C}_{a c} \mathrm{e}^{i\left(\mathcal{S e}_{0}^{\times}+V_{c}^{\times}\right)\left(t_{2}-t_{1}\right)} \times \\
\cdots \\
\times \mathcal{C}_{c d} \mathrm{e}^{i\left(x_{0}^{\times}+V_{d}^{\times}\right)\left(t_{3}-t_{2}\right)} \ldots
\end{array}
$$

Comme dans Clauser-Blume les instants auxquels se produisent les chocs sont supposés donnés par une distribution de Poisson (de fréquence caractéristique v). Par contre, la quantité ci-dessus est calculée sans remplacer chaque opérateur $\mathfrak{C}$ par une valeur moyenne.

L'opérateur $(\mathcal{U}(p))_{a v}$ qui entre dans la forme de raie est alors donné par l'équation (13) de [40] :

$$
\begin{array}{r}
(\mathcal{U}(p))_{a v}=\sum_{a b} p_{a}\left(a \mid\left[p-i \mathcal{H}_{0}^{\times}-i \sum_{j} V_{j}^{\times} F_{j}+\right.\right. \\
\left.+v(1-\mathcal{\zeta})]^{-1} \mid b\right),
\end{array}
$$

et le calcul de la forme de raie nécessite l'inversion d'une matrice de dimension $N=(2 I+1)\left(2 I_{g}+1\right) \times$ $(2 S+1)^{2} \times n$.

Ainsi dans le cas paramagnétique où :

$$
\mathscr{H}_{0}=A \mathbf{I} . \mathbf{S} \text { ou } A_{g} \mathbf{I}_{g} . \mathbf{S} .
$$

Dattagupta fait prendre au hamiltonien $V_{j}$ les 6 valeurs $(n=6)$ :

$$
\pm S_{x} H_{x}, \quad \pm S_{y} H_{y}, \quad \pm S_{z} H_{z} \text {. }
$$

$\mathrm{Si}$, cependant, on adopte, pour décrire la relaxation, le modèle R.P.A. de Dattagupta et Blume [12] : $\mathfrak{C}_{a b}=p_{b}$, l'équation (33) est remplacée par ([40] Eqs. (19)-(20)) :

$$
(u(p))_{a v}=\frac{\left(\mathcal{U}^{0}(p+v)\right)_{a v}}{1-v\left(\mathcal{U}^{0}(p+v)\right)_{a v}},
$$

avec

$$
\left(\mathcal{U}^{0}(p+v)\right)_{a v}=\sum_{j=1}^{n} p_{j}\left[p+v-i \mathcal{X}_{0}^{\times}-i V_{j}^{\times}\right]^{-1},
$$

et la matrice à inverser n'a plus comme dimension que

$$
N=(2 I+1)\left(2 I_{g}+1\right)(2 S+1)^{2},
$$

comme dans le cas Clauser-Blume. 
Si la relaxation est très lente $\left(v \ll V_{j}^{x}\right)$ l'on observera un spectre hyperfin avec une structure due à $V_{j}^{\times}$(par exemple, dans le cas paramagnétique, Eqs. (35) (36), une structure Zeeman associée aux termes $S_{x} H_{x}$, etc...).

$\mathrm{Si}$ la relaxation devient plus rapide $\left(v \gg V_{j}^{\times}\right.$, $v \sim \mathcal{J}_{0}^{\times}$) la décomposition due à $V_{j}^{\times}$disparaît, et (cf. [40] Eq. (21)) :

$$
\begin{aligned}
(U(p))_{a v} & =\left[p-i \mathcal{J}_{0}^{\mathrm{x}}+\sum_{j} p_{j} V_{j}^{\times} \frac{1}{p+v-i \mathcal{J U}_{0}^{\mathrm{x}}} V_{j}^{\times}\right]^{-1} \\
& =\left[p-i \mathcal{J}_{0}^{\times}+R(p)\right]^{-1} .
\end{aligned}
$$

Cette expression est équivalente à celle que l'on obtient par la méthode de la résolvante (cf. ci-dessous) lorsque l'approximation du spectre blanc n'est pas valable : la matrice de relaxation

$$
R(p)=\sum_{j} p_{j} V_{j}^{\times} \frac{1}{p+v-i \mathcal{E}_{0}^{\times}} V_{j}^{\times}
$$

qui dépend des fréquences hyperfines par l'intermédiaire de $\mathcal{H}_{0}^{x}$ est l'équivalent de la matrice - $R^{\prime}\left(\omega+i \frac{\Gamma}{2}\right)$, équation(14) de [50] et équation (42') ci-après $\left(\sum_{j} V_{j}^{x} F_{j} \sim \mathcal{H}_{1}^{x}\right)$.

Enfin, la limite rapide $v \gg V_{j}^{\times}, \mathfrak{H}_{0}^{\times}$correspond à l'approximation du spectre blanc :

$$
R(p) \rightarrow-\sum_{j} p_{j}\left(V_{j}^{\times}\right)^{2} / v,
$$

et l'on retrouve les résultats de Clauser-Blume (cf. [40] Eqs. (25)-(27)).

4.4 Remarques. - Aucune des théories exposées cidessus ne permet de traiter les problèmes d'orientation nucléaire (effets de population dans les spectres Mössbauer à très basse température). D'autre part, le raccord entre les fréquences de saut $(\lambda, v)$ des processus stochastiques et les taux de relaxation microscopiques (lorsqu'ils sont définis) n'est pas toujours évident.

Par contre, les traitements stochastiques fournissent une interprétation phénoménologique simple de problèmes très variés (notamment ceux qui mettent en jeu des phénomènes d'activation, etc...).

\section{Calcul des matrices de relaxation par des traite- ments de perturbation.}

MÉTHODE DE LA MATRICE DENSITÉ ET MÉTHODE DE LA RÉSOLVANTE. - Il existe deux types de traitements utilisant :

- soit la méthode «de l'équation de mouvement de la matrice densité " (Hirst (1970) [15], Gonzalez et al. (1974) [6], Hartmann-Boutron et al. (1975) [5], etc...);

- soit la méthode de la résolvante due à Fano (1963) [16] (Gabriel et al. (1968) [17], Afanasev et al. (1974) [18], Dattagupta et al. (1977) [41]).

Avec la première méthode (cf. § 2) on commence par calculer la forme de raie Mössbauer en l'absence de relaxation. On fait apparaître dans $\mathfrak{J}(\omega)$ un ou plusieurs opérateurs $\mathcal{U}_{0}(t)$ qui sont ensuite remplacés par des opérateurs $\overline{\mathcal{U}}(t)=\exp \left[\left(\frac{i}{\hbar} \mathcal{H}_{0}^{\times}+R\right) t\right]$. La matrice de relaxation $R$ est alors calculée séparément (elle est déduite de $S$, elle-même tirée de l'équation de mouvement de la matrice densité $\sigma[23])$. $R$ ne dépend donc pas de la fréquence d'observation $\omega$.

Avec la deuxième méthode - et en négligeant les effets de populations pour simplifier la présentation on écrit que pour une source ou un absorbeur couplés à un réseau quantique :

$$
\begin{aligned}
J(\omega) & \propto \operatorname{Re} \int_{0}^{\infty} \mathrm{d} t \exp \left[\left(i \omega-\frac{\Gamma}{2}\right) t\right] \underset{\substack{a t \\
\text { reseau }}}{\operatorname{Trace}}\left\{\rho \underset{\sim}{T_{L}} \exp \left[\frac{i}{\hbar}\left(\mathcal{H}_{0}^{\times}+\mathcal{H}_{1}^{\times}+\mathcal{H}_{\mathrm{B}}^{\times}\right) t\right] \underset{\sim}{T_{L}^{\dagger}}\right\}, \\
& \propto \operatorname{Re} \operatorname{Trace}_{\substack{a t \\
\text { reseau }}}^{\infty} \rho \underset{\sim}{T_{L}} \frac{-1}{i \omega-\frac{\Gamma}{2}+\frac{i}{\hbar}\left(\mathcal{H}_{0}^{\times}+\mathcal{H}_{1}^{\times}+\mathcal{H}_{\mathrm{B}}^{\times}\right)} T_{L}^{+},
\end{aligned}
$$

où $\rho$ est la matrice densité du système total (atome + réseau), $\mathfrak{H}_{0}$ le hamiltonien de l'atome, $\mathfrak{H}_{\mathbf{B}}$ celui du réseau et $\mathfrak{H e}_{1}$ le hamiltonien de couplage atome-réseau responsable de la relaxation.

$$
\begin{gathered}
\text { L'opérateur }(-1) /\left[i \omega-\frac{\Gamma}{2}+\left(\frac{i}{\hbar}\right)\left(\mathcal{H}_{0}^{\times}+\mathscr{H}_{1}^{\times}+\mathcal{H}_{\mathrm{B}}^{\times}\right)\right] \text {est ensuite développé en puissances du paramètre : } \\
\frac{-1}{i \omega-\frac{\Gamma}{2}+\frac{i}{\hbar}\left(\mathfrak{H}_{0}^{\times}+\mathcal{H}_{\mathrm{B}}^{\times}\right)} \mathcal{H}_{1}^{\times},
\end{gathered}
$$

et soumis à un certain nombre de manipulations algébriques suivies d'une trace sur les variables du réseau. Finalement $J(\omega)$ prend la forme :

$$
J(\omega) \propto \operatorname{Re} \operatorname{Trace}_{a t}\left\{\underset{\sim}{T} \frac{-1}{i \omega-\frac{\Gamma}{2}+\frac{i}{\hbar} \mathfrak{H}_{0}^{\times}+R^{\prime}\left(\omega+i \frac{\Gamma}{2}\right)} \underset{\sim}{T_{L}^{+}}\right\}
$$


où la matrice de relaxation $R^{\prime}(\omega+i \Gamma / 2)$, qui dépend de la pulsation d'observation $\omega\left(^{6}\right),\left({ }^{7}\right)$ est donnée à l'ordre deux en $\mathcal{H}_{1}^{\times}$par les équations (17), (18), (21) de [41]; mais la dernière de ces équations n'est en fait valable qu'à haute température, car la non-commutation des opérateurs $F_{q^{\prime}}(\tau), F_{q}(0)$ d'un réseau quantique (cf. Eqs. (41') (41") ci-dessous) n'a pas été prise en compte. La démonstration que nous venons de résumer est celle de Dattagupta et al. [41]. Afanasev et al. [18] utilisent pour leur part des opérateurs de projection qui compliquent la présentation.

On remarque que l'équation (40') est identique à l'équation (13), au remplacement près de $R$ par $R^{\prime}(\omega+$ $i \Gamma / 2)$. Les éléments de matrice de $R$ (matrice densité) et $R^{\prime}(\omega+i \Gamma / 2)$ (résolvante) en fonction des densités spectrales sont donnés pour un réseau quantique à température quelconque, sans négliger les déplacements de fréquence (et avec des notations unifiées !), dans la référence [51] : équations (5)-(8) et (12)-(14) : nous les reproduisons brièvement ici en renvoyant le lecteur à cette référence pour des explications plus détaillées.

Supposons que le hamiltonien de relaxation $\varkappa_{1}$ est de la forme :

$$
\mathcal{H}_{1}=\sum_{q} K^{q} F^{q}
$$

où $K^{q}$ est un opérateur atomique et $F^{q}$ un opérateur du réseau, et que les densités spectrales du réseau sont définies en fonction des $F^{q}$ par :

$$
\begin{aligned}
& I_{q^{\prime} q}^{\prime}(\omega)=\frac{1}{\hbar^{2}} \int_{0}^{+\infty} \mathrm{d} \tau \mathrm{e}^{i \omega \tau} \underset{\text { reseau }}{\operatorname{Trace}}\left\{\rho_{\mathrm{B}} F^{q^{\prime}}(\tau) F^{q}(0)\right\} \\
& I_{q^{\prime} q}^{\prime \prime}(\omega)=\frac{1}{\hbar^{2}} \int_{0}^{+\infty} \mathrm{d} \tau \mathrm{e}^{i \omega \tau} \underset{\text { reseau }}{\operatorname{Trace}}\left\{\rho_{\mathrm{B}} F^{q}(0) F^{q^{\prime}}(\tau)\right\},
\end{aligned}
$$

$\left(\rho_{\mathrm{B}}\right.$, matrice densité du réseau; $F^{q^{\prime}}(\tau)$, opérateur du réseau en représentation d'Heisenberg par rapport au hamiltonien du réseau, cf. [2] p. 303). Par rapport aux états propres de $\mathcal{H}_{0}^{\times}$, les éléments de $R$ sont égaux à :

$$
\begin{aligned}
\left\langle g f|R| g^{\prime} f^{\prime}\right\rangle=\left\langle f^{\prime} g^{\prime}|S| f g\right\rangle=\sum_{q q^{\prime}}[ & -\sum_{f^{\prime \prime}} K_{f^{\prime} f^{\prime \prime}}^{q^{\prime}} K_{f^{\prime \prime} f}^{q} I_{q^{\prime} q}^{\prime}\left(\omega_{f f^{\prime \prime}}\right) \delta_{g g^{\prime}} \\
& -\sum_{g^{\prime \prime}} K_{g g^{\prime \prime}}^{q} K_{g^{\prime \prime} g^{\prime}}^{q^{\prime}} I_{q^{\prime} q}^{\prime \prime}\left(\omega_{g^{\prime \prime} g}\right) \delta_{f f^{\prime}} \\
& +K_{f^{\prime} f}^{q^{\prime}} K_{g g^{\prime}}^{q} I_{q^{\prime} q}^{\prime \prime}\left(\omega_{g^{\prime} g}\right) \\
& \left.+K_{f^{\prime} f}^{q} K_{g g^{\prime}}^{q^{\prime}} I_{q^{\prime} q}^{\prime}\left(\omega_{f f^{\prime}}\right)\right]
\end{aligned}
$$

et ceux de $R^{\prime}$ à :

$$
\begin{aligned}
\left\langle g f\left|R^{\prime}\left(\omega+i \frac{\Gamma}{2}\right)\right| g^{\prime} f^{\prime}\right\rangle \equiv & \left\langle f^{\prime} g^{\prime}\left|S^{\prime}\left(\omega+i \frac{\Gamma}{2}\right)\right| f g\right\rangle \\
\equiv \sum_{q q^{\prime}}[ & -\sum_{f^{\prime \prime}} \delta_{g g^{\prime}} K_{f^{\prime} f^{\prime \prime}}^{q^{\prime}} K_{f^{\prime \prime} f}^{q} I_{q^{\prime} q}^{\prime}\left(\omega+\omega_{g^{\prime} f^{\prime \prime}}+i \frac{\Gamma}{2}\right) \\
& -\sum_{g^{\prime \prime}} \delta_{f f^{\prime}} K_{g g^{\prime \prime}}^{q} K_{g^{\prime \prime} g^{\prime}}^{q^{\prime}} I_{q^{\prime} q}^{\prime \prime}\left(\omega+\omega_{g^{\prime \prime} f}+i \frac{\Gamma}{2}\right) \\
& +\sum_{q q^{\prime}} K_{f^{\prime} f}^{q^{\prime}} K_{g g^{\prime}}^{q} I_{q^{\prime} q}^{\prime \prime}\left(\omega+\omega_{g^{\prime} f}+i \frac{\Gamma}{2}\right) \\
& \left.+\sum_{q q^{\prime}} K_{g g^{\prime}}^{q^{\prime}}, K_{f^{\prime} f}^{q} I_{q^{\prime} q}^{\prime}\left(\omega+\omega_{g f^{\prime}}+i \frac{\Gamma}{2}\right)\right]
\end{aligned}
$$

(') $R^{\prime}$ dépend également de $\Gamma$ car le temps de séjour $\tau_{n}$ dans l'état I joue le rôle d'un temps de corrélation pour la relaxation. Dans la pratique, $\Gamma$ joue peu car $\tau_{\mathrm{c}} \ll \tau_{\mathrm{n}}$.

( $\left.{ }^{7}\right)$ Dans [41] Dattagupta emploie la notation $\Gamma$ au lieu de $\Gamma / 2$. 
(où la matrice $S^{\prime}$ est l'homologue de $S$ dans la méthode de type résolvante).

Lorsque l'approximation du spectre blanc est valable les arguments des densités spectrales $I^{\prime}$ et $I^{\prime \prime}$ dans (42) et (42') peuvent être remplacés par 0 et l'on a évidemment $R \equiv R^{\prime}, S \equiv S^{\prime}$.

Lorsque la W.N.A. n'est pas satisfaite et que dans $R^{\prime}$ (Eq. (42')), on remplace $\omega$ par $\omega_{f g}$ et $\Gamma$ par 0 , les arguments des $I^{\prime}, I^{\prime \prime}$ deviennent identiques à ceux des $I^{\prime}, I^{\prime \prime}$ contenus dans $R(\mathrm{Eq}$. (42)) d'où l'on tire :

$$
\begin{aligned}
& \left\langle g f|R| g^{\prime} f^{\prime}\right\rangle= \\
& =\left(\left\langle g f\left|R^{\prime}\left(\omega+i \frac{\Gamma}{2}\right)\right| g^{\prime} f^{\prime}\right\rangle\right)_{\substack{\omega=\omega g g \\
\Gamma \rightarrow 0}}
\end{aligned}
$$

Mais, par ailleurs, en l'absence de W.N.A. les expressions de $R$ et $R^{\prime}$ ci-dessus ne sont applicables qu'à des effets de relaxation petits par rapport à la distance entre raies (condition de validité du traitement de perturbation, cf. discussion dans [1]). Compte tenu de ceci et de la relation (43), on prévoit donc que les formes de raie calculées, soit par la méthode de la matrice densité, soit par la méthode de la résolvante, vont être très voisines, la méthode de la résolvante étant cependant plus élaborée puisque la fréquence d'observation est introduite dans le problème dès le départ. En particulier, lorsque les expériences Mössbauer ont une haute précision, la méthode de la résolvante présente l'avantage de donner accès (par inversion de la forme de raie) à la dépendance en fréquence des densités spectrales $I(\omega)$, d'où l'on peut tirer des informations sur la fonction de corrélation de $\mathcal{H}_{1}$, donc sur la dynamique du processus de relaxation (cf. Eq. (65) ci-dessous).

Bien entendu, les équations (42) (42') (43) restent valables lorsque les $F^{q}$ sont des fonctions fluctuantes classiques; dans ce cas :

$$
I_{q^{\prime} q}^{\prime}(\omega)=I_{q^{\prime} q}^{\prime \prime}(\omega)=I(\omega)=\frac{1}{\hbar^{2}} \int_{0}^{\infty} \mathrm{d} \tau \mathrm{e}^{i \omega \tau} \overline{F^{q^{\prime}}(0) F^{q}(-\tau)}
$$

où la barre désigne une moyenne temporelle.

Notons que, contrairement à ce qui avait été fait dans [1], les densités spectrales (41) et (44) sont définies par des intégrales de 0 à $\infty$, c'est-à-dire sans négliger les déplacements de fréquence; autrement, il suffirait de les réduire à leur partie réelle. En fait, les déplacements de fréquence ne sont non nuls que lorsque l'approximation du spectre blanc n'est pas valable (cf. [2] p. 310 Eq. (IV.37)) et même alors, il ne semble pas utile de les prendre en compte si la précision expérimentale n'est pas excellente.

Dans la pratique, les calculs de perturbation ne s'appliquent qu'aux cas où le hamiltonien $\mathfrak{H}_{1}$ est bien connu, donc essentiellement à la relaxation électronique.

REVUE DE PHYSIQUe APPLIQUÉE. - T. 18, № 7, JUILLET 1983

\section{Traitements de type matrice densité.}

Les plus simples ( $(1$ et 2$)$ sont très analogues à ceux qu'on utilise en R.M.N. mais présentent l'inconvénient de ne pas permettre la description de la zone brouillée. Cette difficulté a été résolue en 1970 par L. L. Hirst, malheureusement au prix d'un gros alourdissement de l'algèbre.

6.1 Relaxation RAPIDE EN PRÉSENCE D'UN EFFET QUADRUPOLAIRE OU ZEEMAN STATIQUE. - Ces calculs (Bradford et Marshall (1966), Wegener (1965), Afanasev et Gorobchenko (1974)) s'appliquent à un spectre résolu dont les raies sont légèrement élargies par la relaxation. Ils sont faits dans l'approximation du spectre blanc. Les formules relatives au cas Zeeman, en présence de relaxation par un champ fluctuant isotrope ou anisotrope, sont données par Litterst et al. (1979) [52] équation (3.6) (N.B. : dans cette référence $\delta \Gamma$ représente la variation de la largeur totale à mi-hauteur). Elles ont fait l'objet de belles vérifications expérimentales dans la thèse de Mørup (1980) [57].

Elles ont également été employées par Afanasev et al. $(1978,1979)$ [46] [52], au cours de leur étude de la réorientation des populations à basse température dans les sources $\underline{\mathrm{Pd}}^{57} \mathrm{Co}$ où se produit la cascade :

$$
\begin{aligned}
& { }^{57} \mathrm{Co}(I=7 / 2) \stackrel{\text { C.E. }}{\longrightarrow}{ }^{57} \mathrm{Fe}(I=5 / 2) 123 \mathrm{keV} \stackrel{\gamma}{\longrightarrow} \\
& \quad \rightarrow{ }^{57} \mathrm{Fe}(I=3 / 2) 14 \mathrm{keV} \underset{\text { Mössbauer }}{\longrightarrow}{ }^{57} \mathrm{Fe}(I=1 / 2) .
\end{aligned}
$$

Ce travail avait pour but d'obtenir des informations sur les populations hyperfines de ${ }^{57} \mathrm{Co}$ (et donc sur un éventuel effet Kondo au niveau du cobalt) en mesurant les anomalies d'intensité et de largeur des raies Mössbauer issues de $I=3 / 2$ et en remontant (théoriquement) la cascade.

Pour ceci, les auteurs ont développé les populations $p_{m}(I)$ des différents états de la cascade en opérateurs tensoriels irréductibles, soit avec les notations de [1] :

$$
\sigma_{k}^{0}(I)=\sum_{m}\left\langle m\left|T_{k}^{0}\right| m\right\rangle p_{m}(I)
$$

L'émission spontanée entre niveaux $I$ ne couple entre eux que les $\sigma_{k}^{0}(I)$ de même $k$ (cf. [5] Eq. (56)). D'autre part, lorsque la température n'est pas trop basse, à l'intérieur de chaque niveau $I$ les relaxations des $\sigma_{k}^{0}(I)$ de $k$ différents sont découplées. Leurs valeurs moyennes sont solutions d'équations de bilan détaillé de la forme (cf. Eq. $\left.\left(15^{\prime \prime}\right)\right)$ :

$$
\begin{aligned}
0 \equiv \frac{\mathrm{d} \sigma_{k}^{0}(I)}{\mathrm{d} t}=\Gamma_{k}\left(I^{\prime}\right. & \rightarrow I) \sigma_{k}^{0}\left(I^{\prime}\right)-\Gamma_{k}\left(I \rightarrow I^{\prime \prime}\right) \times \\
& \times \sigma_{k}^{0}(I)-\frac{k(k+1)}{2 T_{1 n}(I)} \sigma_{k}^{0}(I),
\end{aligned}
$$

où le premier terme représente l'alimentation radiative de $I$ par le niveau supérieur $I^{\prime}$, le deuxième sa 
désexcitation radiative vers le niveau inférieur $I^{\prime \prime}$ et le troisième la relaxation de $\sigma_{k}^{0}(I)\left({ }^{8}\right)$ (cf. [1] Eqs. (114)(115)). On en tire (cf. [52] Eq. (3.22)) :

$$
\sigma_{k}^{0}(I)=\frac{\left[\Gamma_{k}\left(I^{\prime} \rightarrow I\right) / \Gamma_{k}\left(I \rightarrow I^{\prime \prime}\right)\right] \sigma_{k}^{0}\left(I^{\prime}\right)}{1+\frac{k(k+1)}{2 T_{1 n}(I) \Gamma_{k}\left(I \rightarrow I^{\prime \prime}\right)}} .
$$

Ces équations permettent de relier les populations de ${ }^{57} \mathrm{Co}(I=7 / 2)$ à celles de ${ }^{57} \mathrm{Fe}(I=3 / 2)$. Dans ce dernier état : $k \leqslant 2 I=3$, donc seules les composantes $\sigma_{k}^{0}$ de $k \leqslant 3$ des états supérieurs pourront être atteintes. Leur calcul exige la connaissance à la fois des $\sigma_{k}^{0}(I=3 / 2)$ (qu'on tire des intensités intégrées des raies Mössbauer) et de $1 / T_{1 n}(I=3 / 2)$. Ce dernier est déduit de l'élargissement des raies internes du spectre qui - compte tenu de l'équation 3.6 de [52] et des facteurs gyromagnétiques - est essentiellement déterminé par le paramètre $\gamma_{\perp}\left(\propto 1 / T_{1 n}\right)$ de cette référence (au contraire l'élargissement des raies externes dépend surtout du paramètre $\gamma_{\|} \propto 1 / T_{2 n}^{\prime}\left({ }^{9}\right)$ ). Des détails complémentaires sur cette expérience sont donnés dans les articles originaux et dans la revue d'Imbert sur les sources.

\subsection{Relaxation paramagnétiQue. Spectre Réduit} À UNE RAIE UNIQUE DANS LA LIMITE DU RÉTRÉCISSEMENT EXTRÊME. - Supposons que le noyau est uniquement soumis à un hamiltonien hyperfin $\mathscr{H}_{1}(t)=A \mathbf{I} . \mathbf{S}(t)$ dans l'état Mössbauer excité et $\mathscr{H}_{1}(t)=A \mathbf{I}_{g} . \mathbf{S}(t)$ dans l'état fondamental. Si $\mathbf{S}$ ne fluctue pas du tout, on observera une structure hyperfine de type ion libre $\left(\mathbf{F}=\mathbf{I}+\mathbf{S}, \mathbf{G}=\mathbf{I}_{g}+\mathbf{S}\right.$, avec des transitions entre niveaux $F$ et $G$, cf. ${ }^{170} \mathrm{YbAu}$ [8]). $\mathrm{Si}$, au contraire, $S$ fluctue très vite $\left((A / \hbar) T_{1 s} \ll 1\right.$, où $T_{1 s}$ est le temps de relaxation de $S$ ) le spin nucléaire ne peut plus « suivre " le spin électronique, toute trace de structure hyperfine disparaît (" rétrécissement extrême") et le spectre se réduit à une raie unique de demi-largeur $\Gamma / 2+\delta$ avec (Hartmann-Boutron 1980) [55] :

$$
\begin{gathered}
\delta=\frac{S(S+1)}{3 \hbar^{2}} T_{1 s}\left\{A^{2} I(I+1)+A_{g}^{2} I_{g}\left(I_{g}+1\right)-\right. \\
\left.-A A_{g}\left[I(I+1)+I_{g}\left(I_{g}+1\right)-L(L+1)\right]\right\}, \quad(46)
\end{gathered}
$$

où $L$ est l'ordre multipolaire de la transition Mössbauer. Pour ${ }^{57} \mathrm{Fe}\left(I=3 / 2, I_{g}=1 / 2, L=1\right)$ cette expression redonne bien le résultat de Bradford et Marshall (1966). Elle a été appliquée récemment par B. D. Dunlap et al. (1979) à l'interprétation d'expériences sur ${ }^{151} \mathrm{Eu}$.

${ }^{8}$ ) La relaxation est supposée due à un champ fluctuant. $T_{1 n}(I)$ dépend de $I$ de façon connue par l'intermédiaire du facteur gyromagnétique, cf. equation (28) de [1].

(9) Cf. équation (33) de [1].
La condition de validité de la théorie de perturbation $\left(\left\langle\mathscr{H}_{1}^{2}\right\rangle \tau_{\mathrm{c}}^{2} / \hbar^{2} \ll 1\right)$ s'écrit ici $\left(\mathscr{H}_{1} \sim A, \tau_{\mathrm{c}} \sim T_{1 s}\right)$ :

$$
\frac{A}{\hbar} T_{1 s} \ll 1
$$

Le modèle ci-dessus ne permet donc pas de décrire le passage de la raie unique à la structure hyperfine résolue, car la zone brouillée intermédiaire correspond précisément à $\frac{A}{\hbar} T_{1 s} \sim 1$ (soit, dans ${ }^{170} \mathrm{YbAu}$ où $A / \dot{h} \sim 900 \mathrm{MHz}$, à $\left.T_{1 s} \sim 10^{-10} \mathrm{~s}\right)$.

6.3 Relaxation paramagnétiQue. Modèle de Hirst (SPECTRES À hAUTE TEMPÉRATURE). - Pour tourner la difficulté, Hirst (1970) [15] a été amené à introduire un hamiltonien plus compliqué (cf. Clauser-Blume [9]) :

$$
\mathscr{H}=\mathscr{H}_{0}+\mathscr{H}_{1}(t)=A \mathbf{I} . \mathbf{S}-g \mu_{\mathbf{B}} \mathbf{S . H}(t),
$$

où $\mathbf{H}(t)$ est un champ fluctuant responsable de la relaxation électronique. L'intérêt de ce modèle est que le temps de corrélation $\tau_{\mathrm{c}}$ de $\mathcal{H}_{1}(t)$ est maintenant celui du champ fluctuant $\mathbf{H}(t)$ et non plus $T_{1 s}$. $\mathrm{Si}$

$$
\frac{1}{\tau_{\mathrm{c}}} \gg \frac{A}{\hbar} \sim \frac{1}{T_{1 s}} \sim\left\langle\frac{\mathscr{H}_{1}^{2}}{\hbar^{2}}\right\rangle \tau_{\mathrm{c}}
$$

l'approximation du spectre blanc est valable, la théorie de perturbation aussi et il est possible de décrire la zone brouillée $\frac{A}{\hbar} T_{1 s} \sim 1$. Il faut, pour ceci, que $\tau_{\mathrm{c}} \ll 10^{-10} \mathrm{~s}$, condition qui, dans les solides, est satisfaite par de nombreux processus (possédant des $\tau_{\mathrm{c}}$ de l'ordre de $10^{-12}-10^{-13} \mathrm{~s}$ ).

Il en est ainsi dans les métaux, pour la relaxation par les électrons de conduction qui a été traitée d'abord par Hirst (1970) [15] en relation avec ${ }^{166} \mathrm{ErZr}$, puis par Gonzalez et al. (1974) [8] en relation avec ${ }^{170} \mathrm{YbAu}$. Rappelons que la transition Mössbauer de ${ }^{170} \mathrm{Yb}$ $\left(\ll 2^{+} 0^{+} »\right)$ se fait entre deux niveaux $I=2, I_{g}=0$ et que, d'autre part, le niveau électronique le plus bas de $\mathrm{Yb}^{3+}$ dans l'or est un doublet de Kramers cubique $\Gamma_{7}$, de spin effectif $S=1 / 2$ qui, dans l'état Mössbauer excité, possède une structure hyperfine isotrope : $\mathscr{H}_{0}=A I$.S, donnant deux niveaux hyperfins $(\mathbf{F}=$ I + S) $: F=5 / 2$ d'énergie $E=A$ et $F=3 / 2$ d'énergie $E=-3 A / 2$, tandis que le niveau hyperfin fondamental est un doublet dégénéré $G=S=1 / 2$. Le calcul de la forme de raie (Eq. (13)) nécessite l'inversion d'une matrice $20 \times 20$ (qui se factorise en sousmatrices de dimensions $\leqslant 4)$. Gonzalez et al. ont ainsi obtenu une expression analytique ([6] Eqs. (76)(77)) valable pour un champ fluctuant général en symétrie uniaxe et en symétrie cubique, avec ou sans champ appliqué. Dans le cas cubique et en l'absence de champ appliqué, une expression équivalente, mais beaucoup plus simple, a été établie ultérieurement par 
Chopin et al. (1976) [30] à l'aide des opérateurs tensoriels irréductibles. Elle est valable pour n'importe quel champ fluctuant isotrope satisfaisant à l'approximation du spectre blanc :

$$
\begin{gathered}
J(\omega) \propto \operatorname{Re} \frac{p-i \frac{A}{2 \hbar}+\frac{1}{T_{1 s}}}{p^{2}+p\left(\frac{1}{T_{1 s}}-\frac{i A}{2 \hbar}\right)+\frac{3 A^{2}}{2 \hbar^{2}}} \\
\left\{\begin{array}{l}
A / k_{\mathrm{B}} T \ll 1 \\
I=2, \quad I_{g}=0, \quad S=1 / 2,
\end{array}\right.
\end{gathered}
$$

où $p=\frac{\Gamma}{2}-i \omega, A$ est le couplage hyperfin dans l'état Mössbauer excité et $T_{1 s}$ est le temps de relaxation qu'aurait le spin $\mathbf{S}$ en l'absence de structure hyperfine. Comme cela a été dit dans le chapitre précédent, l'équation (48) peut aussi être obtenue par la méthode de Scherer Blume [30]. Lorsque la relaxation est lente $\left(1 / T_{1 \text { s }}\right.$ petit), l'équation (48) fournit un spectre de deux raies correspondant aux transitions issues des deux niveaux hyperfins $F=5 / 2$ et $F=3 / 2$. Quand $1 / T_{1 s}$ croît, le spectre se brouille et lorsque $1 / T_{1 s} \gg A$ le spectre se réduit à une raie unique; tout ceci en accord avec l'expérience. Dans un métal la relaxation est due aux électrons de conduction et $1 / T_{1 s}=C_{K} T$ (loi de Korringa) ([6] Eq. (82)). Par ailleurs, en présence d'un effet Kondo tel que $T_{K} \ll T$, on démontre que l'équation (48) reste valable à condition d'introduire dans $1 / T_{1 s}$ une correction Kondo en $\log T: 1 / T_{1 s}=$ $C_{K} T(1+b \log T)$ (cf. [7] Eq. (28)).

La référence [30] contient également (Eqs. (40)-(47)) les formules nécessaires au calcul de la forme de raie Mössbauer correspondant à des $I, I_{g}$ et $S$ quelconques et à un champ fluctuant isotrope satisfaisant à l'approximation du spectre blanc. Elles supposent que le hamiltonien hyperfin (magnétique, quadrupolaire) est isotrope (ce qui n'est pas toujours réalisé dans les solides lorsque le spin effectif $S$ est différent de $1 / 2$ cf. [30]), et n'est pas en compétition avec un hamiltonien de spin : $a\left(S_{x}^{4}+S_{y}^{4}+S_{z}^{4} \ldots\right)$ de grandeur comparable (cela peut se produire pour les ions $« S »$ tels que $\left.\mathrm{Fe}^{3+}\right)$. $\mathfrak{J}(\omega)$ s'obtient alors par inversion d'une matrice de dimension égale au nombre de couples $F G$ $\left(\mathbf{F}=\mathbf{I}+\mathbf{S}, \mathbf{G}=\mathbf{I}_{g}+\mathbf{S}\right)$. Ainsi, si $S=1 / 2$ et $I, I_{g}$ sont arbitraires, il y. a deux niveaux $F$ et deux niveaux $G$ et cette matrice est $4 \times 4$, sauf si $I_{g}=0$ (un seul niveau $G$ ), auquel cas elle est $2 \times 2$ (au lieu de $20 \times 20$ pour la supermatrice complète correspondant à $\left.{ }^{170} \mathrm{YbAu}\right)$. Dans le cas de ${ }^{57} \mathrm{Fe}^{3+}: S=5 / 2, I=$ $3 / 2, I_{g}=1 / 2$, ce qui donne 4 niveaux $F$ et 2 niveaux $G$ et la matrice à diagonaliser est $8 \times 8$ (au lieu de $288 \times 288$ pour la supermatrice complète).

La matrice en question est la sous-matrice de la supermatrice $i \omega-\frac{\Gamma}{2}-\frac{i}{\hbar} \mathcal{H}_{0}^{\times}+S$ qui caractérise l'évolution des composantes tensorielles d'ordre $L$ associées aux multipôles ${ }^{F G} V_{L}^{M}$ responsables de la transition Mössbauer $\left({ }^{10}\right)$. Par rapport à une base $\mid F G>$ elle a pour éléments :

$$
\left\langle L, F^{\prime}, G^{\prime}\left|i \omega-\frac{\Gamma}{2}-\frac{i}{\hbar} \mathcal{H}_{0}^{\times}+S\right| L, F G\right\rangle=\delta_{F F^{\prime}} \delta_{G^{\prime}}\left(i \omega-\frac{\Gamma}{2}-i \omega_{F G}\right)-\beta_{L}^{\prime}\left(F G \leftrightarrow F^{\prime} G^{\prime}\right),
$$

où le coefficient de relaxation $\beta_{L}^{\prime}$ est donné par l'équation (44) de [30], en fonction de $L$ et de l'ordre tensoriel $l$ du processus de relaxation. Pour un champ fluctuant $(l=1)$ :

$$
\begin{aligned}
& \beta_{L}^{\prime}\left(F^{\prime} G^{\prime} \leftrightarrow F G\right)=\frac{S(S+1)}{T_{1 s}} \times \\
& \times\left[\delta_{F F^{\prime}} \delta_{G G^{\prime}} \cdot+(-1)^{I_{g}-I}(-1)^{F+F^{\prime}+L}(2 S+1) \sqrt{(2 F+1)\left(2 F^{\prime}+1\right)(2 G+1)\left(2 G^{\prime}+1\right)}\right. \\
& \left.\times\left\{\begin{array}{ccc}
G^{\prime} & G & 1 \\
S & S & I_{g}
\end{array}\right\}\left\{\begin{array}{ccc}
F^{\prime} & F & 1 \\
S & S & I
\end{array}\right\}\left\{\begin{array}{ccc}
F & G & L \\
G^{\prime} & F^{\prime} & 1
\end{array}\right\}\right]
\end{aligned}
$$

(où $T_{1 s}$ est le temps de relaxation qu'aurait le spin $S$ en l'absence de couplage hyperfin).

Après inversion, la forme de raie s'obtient immédiatement à l'aide des équations (43) et (37) de [30] :

$$
J(\omega) \propto \sum_{\substack{F G \\ F^{\prime} G^{\prime}}} b_{L}^{F^{\prime} G^{\prime}} b_{L}^{F G}\left\langle L, F^{\prime} G^{\prime}\left|\frac{-1}{i \omega-\frac{\Gamma}{2}-\frac{i}{\hbar} J \mathcal{H}_{0}^{\times}+S}\right| L, F G\right\rangle,
$$

(19) En présence de couplage hyperfin, on ne peut pas raisonner sur les opérateurs $I_{g} T_{L}^{M}$ de l'équation (22). Il faut introduire de nouveaux opérateurs ${ }^{F G} V_{L}^{M}$ adaptés au système couplé, qui sont reliés aux $I_{g}^{g} T_{L}^{M}$ par des coefficients « $6 \mathrm{j}$ ». Ceci explique la lourdeur de l'algèbre qui conduit aux équations (49) (50). Dans la référence [30], nous avons considéré l'évolution des composantes tensorielles ${ }^{F G} \sigma_{L}^{M}$ (off-diagonales entre $I$ et $I_{g}$ ) de la matrice densité $\sigma$, au lieu de raisonner directement sur les ${ }^{F G} V_{L}^{M}(t)$ comme dans [51] [56]. Les deux méthodes sont équivalentes. 
avec

$$
b_{L}^{F G}=(-1)^{I+S+G+L} \sqrt{(2 F+1)(2 G+1)}\left\{\begin{array}{lll}
F & G & L \\
I_{g} & I & S
\end{array}\right\}
$$

Dans ces équations, les \{\} sont des coefficients « $6 \mathrm{j} »$, dont il existe des tables (M. Rotenberg et al. « The $3 \mathrm{j}$ and 6j symbols »). Plus récemment, Hartmann-Boutron 1980 [56], la méthode de Hirst a été étendue au cas où l'approximation du spectre blanc n'est pas satisfaite (parce que $1 / \tau_{\mathrm{c}} \sim A / \hbar$, ou pour des raisons plus subtiles, cf. ci-dessous). Malheureusement, les expressions que l'on obtient alors ne peuvent être appliquées qu'à un spectre hyperfin résolu dont les raies sont un peu élargies par la relaxation (effets de relaxation petits par rapport à la distance entre raies). Dans le cas $I=2, I_{g}=0, S=1 / 2$, où l'état Mössbauer excité est décomposé en deux niveaux hyperfins, $F=5 / 2$ d'énergie $E=+A$ et $F=3 / 2$ d'énergie $E=-3 / 2 A$, et lorsqu'on néglige les déplacements de fréquence, la forme de raie (48) pour la relaxation par un champ fluctuant reste valable, mais avec un $T_{1 s}$ qui dépend de la pulsation hyperfine $5 / 2(A / \hbar)([56]$ Eqs. (11)(12)) :

$$
\frac{1}{T_{1 s}}=\operatorname{Re} I(0)+\operatorname{Re} I\left(\frac{5}{2}\left|\frac{A}{\hbar}\right|\right)
$$

avec :

$$
I(\omega)=\frac{g^{2} \mu_{\mathrm{B}}^{2}}{\hbar^{2}} \int_{0}^{\infty} \mathrm{d} t \mathrm{e}^{i \omega t}\left\langle H_{z}(0) H_{z}(t)\right\rangle .
$$

Lorsque $I, I_{g}, S$ sont quelconques, la forme de raie s'obtient comme précédemment (cf. Eq. (51)) par inversion d'une matrice dont les éléments par rapport à une base $|G F>o u| F G>$ s'écrivent :

$$
\begin{aligned}
\left\langle L, G F\left|i \omega-\frac{\Gamma}{2}+\frac{i}{\hbar} \mathcal{H}_{0}^{\times}+R\right| L, G^{\prime} F^{\prime}\right\rangle & \equiv\left\langle L, F^{\prime} G^{\prime}\left|i \omega-\frac{\Gamma}{2}-\frac{i}{\hbar} \mathcal{H}_{0}^{\times}+S\right| L, F G\right\rangle \\
& =\delta_{G G^{\prime}} \delta_{F F^{\prime}}\left(i \omega-\frac{\Gamma}{2}+i \omega_{G F}\right)+\alpha_{L}\left(G F \leftrightarrow G^{\prime} F^{\prime}\right),
\end{aligned}
$$

où le coefficient de relaxation $\alpha_{L}\left(G F \leftrightarrow G^{\prime} F^{\prime}\right)$ pour un champ fluctuant isotrope est donné par l'équation (1) de [56] :

$$
\begin{aligned}
& \alpha_{L}\left(G F \leftrightarrow G^{\prime} F^{\prime}\right) \equiv \operatorname{Trace}\left\{G F V_{L}^{M \dagger}\left\{R^{G^{\prime} F^{\prime}} V_{L^{\prime}}^{M^{\prime}}\right\}\right\}=-S(S+1) \delta_{L L^{\prime}} \delta_{M M^{\prime}} \times \\
& \times\left[\delta _ { F F ^ { \prime } } \delta _ { G G ^ { \prime } } \left(\sum_{G^{\prime \prime}}(2 S+1)\left(2 G^{\prime \prime}+1\right)\left\{\begin{array}{ccc}
S & G^{\prime \prime} & I_{g} \\
G & S & 1
\end{array}\right\}^{2} I\left(\omega_{G^{\prime \prime} G}\right)\right.\right. \\
& \left.+\sum_{F^{\prime \prime}}(2 S+1)\left(2 F^{\prime \prime}+1\right)\left\{\begin{array}{ccc}
S & F^{\prime \prime} & I \\
F & S & 1
\end{array}\right\}^{2} I\left(\omega_{F F^{\prime \prime}}\right)\right) \\
& +(-1)^{I-I_{g}}(-1)^{F+F^{\prime}+L}(2 S+1) \times \sqrt{(2 F+1)\left(2 F^{\prime}+1\right)(2 G+1)\left(2 G^{\prime}+1\right)} \\
& \left.\times\left\{\begin{array}{ccc}
G & G^{\prime} & 1 \\
S & S & I_{g}
\end{array}\right\}\left\{\begin{array}{ccc}
F & F^{\prime} & 1 \\
S & S & I
\end{array}\right\}\left\{\begin{array}{ccc}
G & F & L \\
F^{\prime} & G^{\prime} & 1
\end{array}\right\}\left(I\left(\omega_{F F^{\prime}}\right)+I\left(\omega_{G^{\prime} G}\right)\right)\right],
\end{aligned}
$$

dont l'expression simplifiée correspondant à $I_{g}=0$ se trouve dans [56], equation (3). Si l'on fait l'approximation du spectre blanc, (56) se réduit comme attendu à (49) $\left(1 / T_{1 s}=2 I, \alpha_{L} \rightarrow-\beta_{L}^{\prime}\right)$.

Les formules (48) et (53) sont applicables au calcul des spectres de relaxation de ${ }^{160}$ Dy dans un supraconducteur de première espèce ([56] Eqs. (12), (11), (22)) où l'approximation du spectre blanc n'est pas valable à cause des particularités de la densité d'états des quasi particules (pour plus de détails, voir le paragraphe 2 du chapitre "résolvante" ci-après).
6.4 EFFETS DE RÉORIENTATION DES POPULATIONS DANS LES SOURCES ${ }^{170} \mathrm{YbAu}$ À TRÈS BASSE TEMPÉRATURE. Les expériences sur ${ }^{170} \mathrm{YbAu}$ ont été faites sur des sources, où l'état Mössbauer excité est peuplé par le parent ${ }^{170} \mathrm{Tm}$. A haute température (cf. paragraphe précédent) ces effets de peuplement n'influent pas sur la forme de raie qui est donnée par l'équation (13). Par contre, à basse température $\left(k_{\mathrm{B}} T \lesssim 5 A / 2=0,11 \mathrm{~K}\right)$, il faut utiliser l'équation (14) qui fait intervenir la matrice densité moyenne $\sigma^{I}(1 / \Gamma)$ du niveau Mössbauer émetteur. A ces températures, les spectres se com- 
posent de deux raies correspondant aux deux transitions $F=5 / 2, F=3 / 2 \rightarrow G=1 / 2$, encore légèrement élargies par la relaxation, et la réorientation des populations dans l'état émetteur (redistribution entre les deux niveaux $F$ ) se traduit par des anomalies d'intensités (aires intégrées) de ces raies.

La matrice densité initiale $\sigma_{I}^{\text {in }}$ juste après peuplement par le parent est diagonale. $\sigma_{I}$ le reste-t-elle ? Lorsqu'on étudie l'équation d'évolution de $\sigma_{I}$ en présence de relaxation par les électrons de conduction, il est clair que les populations $: p_{m}^{F} \equiv \sigma_{m m}^{F F}$ ( $m$ valeur propre de $F_{z}$ ) sont découplées des termes non diagonaux du type $\sigma_{m m^{\prime}}^{F F^{\prime}}\left(m \neq m^{\prime}\right)$ par raison de symétrie. Mais cela n'est pas évident pour les termes non diagonaux du type $\sigma_{m m}^{F F^{\prime}}\left(F \neq F^{\prime}\right)$. En 1974, HartmannBoutron [7], nous avions supposé que la relaxation était assez faible $(R \lesssim \Gamma)$ pour que le découplage résulte de l'approximation séculaire (car les $p_{m}^{F}$ oscillent à la pulsation 0 et les $\sigma_{m m}^{F F^{\prime}}$ à $\left.\pm 5 / 2(A / \hbar)\right)$. On peut alors établir que les aires intégrées des deux raies hyperfines sont proportionnelles aux populations moyennes $P_{F}(1 / \Gamma)=\sum_{m} p_{m}^{F}(1 / \Gamma)$ des deux niveaux hyperfins, que l'on obtient par des équations de bilan détaillé (cf. Eq. (15")) dans lesquelles on peut d'ailleurs introduire des corrections Kondo, cf. [8] équations (11) et (24). Ceci permet de tirer des spectres des informations sur la relaxation entre les deux niveaux hyperfins, laquelle suggère l'existence d'un effet Kondo sur $\mathrm{Yb}^{3+}$ (Gonzalez et al. 1974 [8], Bonville et al. (1981) : [60] et à paraître).

Plus récemment (Hartmann-Boutron (1981), non publié) nous avons réussi à démontrer que pour un champ fluctuant isotrope l'évolution des $P_{F}$ restait découplée de celle du reste de la matrice densité $\sigma_{I}$ à toute température. La démonstration est basée sur l'équipopulation initiale des sous-niveaux hyperfins
$|F m\rangle$ par le parent ${ }^{170} \mathrm{Tm}^{3+}$ (dont l'état électronique le plus bas dans l'or est diamagnétique) et sur des règles de somme.

Ceci fait, il est possible d'établir une forme de raie $J(\omega)$ de ${ }^{170} \mathrm{Yb}$ valable à basse température (Bonville (1981) par inversion d'une grosse matrice [60], Hartmann-Boutron (1981) en utilisant les opérateurs tensoriels irréductibles). Quand $k_{\mathrm{B}} T<\frac{5}{2} A$ les matrices $R$ et $S$ ne sont pas symétriques par rapport à $G F$ et $G^{\prime} F^{\prime}$, et $\left\langle L G F|R| L G^{\prime} F^{\prime}\right\rangle$ s'obtient à partir de l'équation (56) en remplaçant les quatre densités spectrales $I$ respectivement par $I^{\prime \prime}, I^{\prime}, I^{\prime}, I^{\prime \prime}$ avec (cf. [56] p. 1295) :

$$
\begin{aligned}
& I^{\prime}(\omega)=\frac{g^{2} \mu_{\mathrm{B}}^{2}}{\hbar^{2}} \int_{0}^{\infty} \mathrm{e}^{i \omega t}\left\langle H_{z}(t) H_{z}(0)\right\rangle \mathrm{d} t \\
& I^{\prime \prime}(\omega)=\frac{g^{2} \mu_{\mathrm{B}}^{2}}{\hbar^{2}} \int_{0}^{\infty} \mathrm{e}^{i \omega t}\left\langle H_{z}(0) H_{z}(t)\right\rangle \mathrm{d} t,
\end{aligned}
$$

où $\mathbf{H}$ est maintenant un champ fluctuant quantique dont les densités spectrales sont définies suivant les équations (41'), (41") (cf. Eqs. (12)-(13) de [51]).

Lorsqu'on ne tient pas compte des déplacements de fréquence, $I^{\prime}(\omega)$ et $I^{\prime \prime}(\omega)$, équation (57) peuvent être remplacées par leurs parties réelles qui satisfont à :

$$
\operatorname{Re} I^{\prime \prime}(-\omega)=\operatorname{Re} I^{\prime}(+\omega) \text {. }
$$

Si l'on pose alors :

$$
\begin{aligned}
u & =\operatorname{Re} I^{\prime}(0) \\
v & =\operatorname{Re} I^{\prime}(5 / 2(A / \hbar)) \\
w & =\operatorname{Re} I^{\prime}(-5 / 2(A / \hbar))
\end{aligned}
$$

la forme de raie s'écrit $\left(I=2, I_{g}=0, S=1 / 2\right.$; $p=\Gamma / 2-i \omega)$ :

$$
J(\omega) \propto \operatorname{Re}\left\{\frac{\left(p+\frac{i A}{\hbar}\right) P_{3 / 2}\left(\frac{1}{\Gamma}\right)+\left(p-\frac{3}{2} \frac{i A}{\hbar}\right) P_{5 / 2}\left(\frac{1}{\Gamma}\right)+u+\frac{2}{5} v+\frac{3}{5} w}{p^{2}-\frac{i A}{2 \hbar} p+\left(u+\frac{2}{5} v+\frac{3}{5} w\right) p+\frac{3 A^{2}}{2 \hbar^{2}}-i \frac{3 A}{5 \hbar}(v-w)}\right\},
$$

dans laquelle : $p=\frac{\Gamma}{2}-i \omega$ et $P_{3 / 2}\left(\frac{1}{\Gamma}\right), P_{5 / 2}\left(\frac{1}{\Gamma}\right)$ sont les populations moyennes des niveaux $F=\frac{3}{2}$ et $F=\frac{5}{2}$, normalisées à :

$$
P_{3 / 2}\left(\frac{1}{\Gamma}\right)+P_{5 / 2}\left(\frac{1}{\Gamma}\right)=1
$$

A haute température $\left(T \gg \Delta / k_{\mathrm{B}}=5 A / 2 k_{\mathrm{B}}=\right.$ $0,11 \mathrm{~K}), \quad \sigma_{I}=1$, d'où $P_{3 / 2}=4 / 10$ et $P_{5 / 2}=6 / 10$ (populations proportionnelles aux poids statistiques des niveaux) et $v=\mathrm{w}$ : on retrouve bien les équations
(48), (53) lorsque l'approximation du spectre blanc n'est pas valable et l'équation (48) lorsqu'elle l'est. Quand $T \lesssim \Delta / k_{\mathrm{B}}$ et que la relaxation est due aux électrons de conduction :

$$
\begin{aligned}
& u=\frac{C_{\mathrm{K}} T}{2} \\
& v=\frac{C_{\mathrm{K}}}{2} \frac{\Delta}{k_{\mathrm{B}}} \frac{\exp \left(\frac{\Delta}{k_{\mathrm{B}} T}\right)}{\exp \left(\frac{\Delta}{k_{\mathrm{B}} T}\right)-1} \\
& w=\frac{C_{\mathrm{K}}}{2} \frac{\Delta}{k_{\mathrm{B}}} \frac{1}{\exp \left(\frac{\Delta}{k_{\mathrm{B}} T}\right)-1},
\end{aligned}
$$


où $C_{\mathrm{K}}$ est la constante de Korringa de haute température (les corrections Kondo ne sont pas prises en compte). Pour ${ }^{170} \mathrm{YbAu}$, il se trouve que les élargissements de raie dus à $\overline{u, v}, w$ ne deviennent importants par rapport à la largeur naturelle qu'au-dessus de $1 \mathrm{~K}$, où l'approximation du spectre blanc devient valable $\left(u=v=w=C_{K} T / 2\right)$. On peut donc penser que la forme de raie (61) est valable à toute température. Il resterait à calculer les corrections Kondo à $u, v, w$, comme cela a déjà été fait pour les $P_{F}(1 / \Gamma)$ ([8] Eq. (24)) et pour $1 / T_{1 s}$ dans la forme de raie à haute température, équation (48) (cf. [7], Eq. (28)).

\section{Traitements de type résolvante.}

Tous les articles de l'école russe (Afanasev et al.) utilisent cette méthode, quitte à faire ensuite l'approximation du spectre blanc sur la matrice $R^{\prime}$ lors de certaines applications (qui, pour cette raison, ont été discutées dans le chapitre précédent). Le présent chapitre est, au contraire, consacré aux cas où la résolvante prend son plein intérêt, c'est-à-dire où l'approximation du spectre blanc n'est pas valable.

7.1 Relaxation DE ${ }^{170} \mathrm{Yb}$ Dans $\mathrm{Cs}_{2} \mathrm{NaYbCl}_{6}$. Dans ce composé cubique, le niveau le plus bas de $\mathrm{Yb}^{3+}$ est un doublet de Kramers isotrope $\Gamma_{6}$ de spin effectif $S=1 / 2$ avec une structure hyperfine $\mathscr{H}_{0}=A \mathbf{I}$.S. Les atomes d'ytterbium sont éloignés les uns des autres et à l'hélium on observe des spectres résolus, mais élargis par la relaxation dipôle-dipôle [36]. Une première étude théorique a été effectuée par Dattagupta et al. (1977) [41] qui remplacent le couplage dipôle-dipôle par un champ fluctuant phénoménologique agissant sur $\mathbf{S}$ (cf. $\S 8.1 .3$ ).

La forme de raie $\phi(v)$ et la matrice de relaxation $R(v)$ sont données par les équations (15)-(18) de [41]. Des expressions explicites de $R(v)$ sont ensuite obtenues en supposant d'abord un hamiltonien de relaxation de la forme $\sum_{q} K^{q} F^{q}(t)$ (Eq. 21), puis des fonctions de corrélation $\left\langle F^{q}(0) F^{q^{\prime}}(t)\right\rangle=\left\langle F^{q} F^{q^{\prime}}\right\rangle \times$ $\mathrm{e}^{-\lambda_{q q} t}$ (Eq. (25)), puis un couplage $\sum_{q} K^{q} F^{q}(t)=$ $\mathbf{S . h}(t)$ avec pour seules fonctions de corrélation non nulles (relaxation isotrope) :

$$
\begin{aligned}
\left\langle h_{x}(0) h_{x}(t)\right\rangle & =\left\langle h_{y}(0) h_{y}(t)\right\rangle=\left\langle h_{z}(0) h_{z}(t)\right\rangle \\
& =\frac{1}{3}\left\langle h^{2}\right\rangle \mathrm{e}^{-v_{c} t}
\end{aligned}
$$

cf. équation (31). Le spectre Mössbauer a été calculé à l'aide des équations (15) et (31) de [41], en champ nul et en présence de champ.

Le raccord entre les notations de Dattagupta et celles de la présente revue a été examiné par HartmannBoutron (1979) [51] : les équations de Dattagupta [41] contiennent $\Gamma$ au lieu de $\Gamma / 2$. Par ailleurs, sa forme de raie (15) pour le rayonnement émis n'est, en fait, valable qu'à haute température où la matrice densité de l'état Mössbauer excité est égale à 1 : en effet, une expression correcte devrait contenir la matrice $\sigma^{I}(1 / \Gamma)$ et non la matrice de Boltzmann $\rho_{0}\left({ }^{11}\right)$. Enfin, comme nous l'avons déjà signalé dans le chapitre 5 , l'équation (21) de [41] n'est, elle aussi, valable qu'à haute température, car Dattagupta a supposé

$$
\overline{F_{q}(t) F_{q^{\prime}}(0)}=\overline{F_{q^{\prime}}(0) F_{q}(t)},
$$

ce qui n'est pas vérifié pour un réseau quantique si $\mathcal{H}_{\mathrm{o}} / k_{\mathrm{B}} T \gtrsim 1$. Par contre (62') est effectivement satisfaite dans le cas pratique traité par Dattagupta, qui est celui d'un champ fluctuant classique, cf. équation (62) ci-dessus.

La relaxation dans $\mathrm{Cs}_{2} \mathrm{NaYbCl}_{6}$ en champ nul a également été étudiée par Afanasev et al. (1978) qui ont traité la relaxation dipôle-dipôle par une méthode de moments [44, 45]. Compte tenu de la symétrie cubique, et pour une poudre, le spectre est donné par les équations (18), (24), (27) de [45] (avec $\bar{\omega}=$ $\omega+i \Gamma / 2)$ :

$$
\begin{gathered}
\phi(\omega)=-\operatorname{Im}\left[\frac{2}{5} F_{1}(\omega)+\frac{3}{5} F_{2}(\omega)\right] \\
F_{1}(\omega)=\frac{\bar{\omega}+\frac{1}{2} A-\gamma_{1}(\omega)}{\bar{\omega}\left(\bar{\omega}+\frac{1}{2} A-\gamma_{1}(\omega)\right)-\frac{3}{2} A^{2}}, \\
F_{2}(\omega)=\frac{\bar{\omega}+\frac{1}{2} A-\gamma_{2}(\omega)}{\bar{\omega}\left(\bar{\omega}+\frac{1}{2} A-\gamma_{2}(\omega)\right)-\frac{3}{2} A^{2}} .
\end{gathered}
$$

Des évaluations de moments permettent ensuite de montrer que dans $\mathrm{Cs}_{2} \mathrm{NaYbCl}_{6}$ les termes de relaxation $\gamma_{1}(\omega)$ et $\gamma_{2}(\omega)$, équations (26)-(28) de [45], sont très peu différents : $\gamma_{1}(\omega) \simeq \gamma_{2}(\omega) \simeq \gamma(\dot{\omega})$ (Eq. 50) où $\gamma(\omega)$ est relié à la fonction d'autocorrélation $K(\omega)$ du spin $\mathbf{S}$ par l'équation :

$$
\begin{aligned}
\gamma(\omega) & \equiv \gamma^{\prime}(\omega)+i \gamma^{\prime \prime}(\omega) \\
& =\Delta \omega^{2}\left[K(\omega-A)+K\left(\omega+\frac{3}{2} A\right)\right],
\end{aligned}
$$

dans laquelle $\Delta \omega^{2}$ (Eq. (36) de [45]) est analogue à un second moment. La forme de raie devient donc :

$$
\phi(\omega)=-\operatorname{Im} \frac{\bar{\omega}+\frac{1}{2} A-\gamma(\omega)}{\bar{\omega}\left(\bar{\omega}+\frac{1}{2} A-\gamma(\omega)\right)-\frac{3 A^{2}}{2}},
$$

et son inversion permet de déterminer $\gamma^{\prime}(\omega)$ et $\gamma^{\prime \prime}(\omega)$.

$\left({ }^{11}\right)$ Le calcul de forme de raie de [41] est (en principe) relatif à une source à basse température ; mais dans la réalité, les expériences sur $\mathrm{Cs}_{2} \mathrm{NaYbCl}_{6}$ sont faites en transmission et à haute température. Il faut alors utiliser l'équation (15) de [41] avec $\rho_{0}=1$ (ce qu'a fait Dattagupta). 
Les valeurs ainsi tirées de l'expérience ont été comparées à des valeurs calculées en utilisant un modèle gaussien pour $K(\omega)$ (Eq. (53) de [45]). L'accord est bon; il peut être encore amélioré si on lève certaines approximations faites dans l'évaluation des moments [66].

La simplicité de l'équation (65) ci-dessus a suggéré à Hartmann-Boutron (1979) [51] que ces approximations devaient revenir à représenter l'effet des interactions dipôle-dipôle par un champ fluctuant isotrope. Le recours aux opérateurs tensoriels irréductibles permet effectivement de montrer que pour un champ fluctuant isotrope (cf. [51] Eq. (62)) :

$$
J(\omega) \propto \operatorname{Re} \frac{p-i \frac{A}{2 \hbar}+(X+Y)}{p^{2}+\left(X+Y-i \frac{A}{2 \hbar}\right) p+\frac{3 A^{2}}{2 \hbar^{2}}}
$$

$$
X+Y=I\left(\omega+\frac{3 A}{2 \hbar}+i \frac{\Gamma}{2}\right)+I\left(\omega-\frac{A}{\hbar}+i \frac{\Gamma}{2}\right)
$$

où $I(\omega)$ est défini par l'équation (54) ci-dessus. Compte tenu des différences de notations $(-i \gamma=X+Y$, cf. [51] p. 70) ces formules sont bien en accord avec celles d'Afanasev et al. Notons que, lorsque dans l'équation (67), on remplace $\Gamma$ par 0 et $\omega$ par $A / \hbar$ ou $-3 / 2 A / \hbar, \operatorname{Re}(X+Y)$ se réduit à $1 / T_{1 s}$ équation (53). Il s'agit là d'une illustration de la relation (43) entre $R$ et $R^{\prime}(\omega+i \Gamma / 2)$.

La référence [51] contient également des équations générales (Eqs. (37)-(40), (54)) qui permettent de calculer $J(\omega)$ quels que soient $I, I_{g}$ et $S$. Comme dans le chapitre précédent, $J(\omega)$ s'obtient à partir des équations (51), (55) dans lesquelles le coefficient de relaxation $\alpha_{L}^{\prime}$ pour un champ fluctuant isotrope est donné par ([51] Eq. (54)) :

$$
\begin{aligned}
\alpha_{L}^{\prime}\left(G F \leftrightarrow G^{\prime} F^{\prime}\right) \equiv \operatorname{Trace} & \left\{{ }^{G} V_{L}^{M^{\dagger}}\left\{R^{\prime}\left(\omega+i \frac{\Gamma}{2}\right) G^{\prime} F^{\prime} V_{L^{\prime}}^{M^{\prime}}\right\}\right\}=-S(S+1) \delta_{L L^{\prime}} \delta_{M M^{\prime}} \times \\
& \times\left[\delta _ { F F ^ { \prime } } \delta _ { G G ^ { \prime } } \left(\sum_{G^{\prime \prime}}(2 S+1)\left(2 G^{\prime \prime}+1\right)\left\{\begin{array}{ccc}
S & G^{\prime \prime} & I_{g} \\
G & S & 1
\end{array}\right\}^{2} I\left(\omega+\omega_{G^{\prime \prime} F}+i \frac{\Gamma}{2}\right)\right.\right. \\
& \left.+\sum_{F^{\prime \prime}}(2 S+1)\left(2 F^{\prime \prime}+1\right)\left\{\begin{array}{ccc}
S & F^{\prime \prime} & I \\
F & S & 1
\end{array}\right\}^{2} I\left(\omega+\omega_{G F^{\prime \prime}}+i \frac{\Gamma}{2}\right)\right) \\
& +(-1)^{I-I_{g}}(-1)^{F+F^{\prime}+L}(2 S+1) \times \sqrt{(2 F+1)\left(2 F^{\prime}+1\right)(2 G+1)\left(2 G^{\prime}+1\right)} \\
& \times\left\{\begin{array}{ccc}
G & G^{\prime} & 1 \\
S & S & I
\end{array}\right\}\left\{\begin{array}{ccc}
F & F^{\prime} & 1 \\
S & S & I
\end{array}\right\}\left\{\begin{array}{ccc}
G & F & L \\
F^{\prime} & G^{\prime} & 1
\end{array}\right\}\left(I\left(\omega+\omega_{G F^{\prime}}+i \frac{\Gamma}{2}\right)\right. \\
& \left.\left.+I\left(\omega+\omega_{G^{\prime} F}+i \frac{\Gamma}{2}\right)\right)\right] .
\end{aligned}
$$

(dont la forme simplifiée pour $I_{g}=0$ est donnée par l'équation (59) de [51]). Ici encore, $\alpha_{L}^{\prime}$ coïncide avec $\alpha_{L}$ (Eq. (56)) chaque fois que $\omega=\omega_{F G}$.

7.2 RelaXATION DE ${ }^{160}$ Dy DANS LE THORIUM SUPRACONDUCTEUR. - La méthode de la résolvante a également été utilisée par Wagner et al. (1981) [63] pour interpréter les spectres de relaxation de ${ }^{160} \mathrm{Dy}$ (transition $2^{+} 0^{+}$comme ${ }^{170} \mathrm{Yb}$ ) dans le thorium, supraconducteur de première espèce, où son niveau électronique le plus bas est un doublet de Kramers cubique $\Gamma_{7}$. Les particularités de ce travail sont discutées dans [56] (Note added in proof) : la principale est que la matrice $R^{\prime}(\omega+i \Gamma / 2)$, limitée à sa partie réelle, y est calculée à l'ordre $\hbar \omega / k_{\mathrm{B}} T \sim \mathscr{H}_{0} / k_{\mathrm{B}} T$. Mais si les termes en $\mathscr{H}_{0} / k_{\mathrm{B}} T$ ne sont pas négligeables, il faudrait en principe, puisqu'il s'agit d'une expérience de source, utiliser l'expression de forme de raie basse température, équation (14), qui contient la matrice densité $\sigma^{I}(1 / \Gamma)$ (cf. Eq. (69) ci-après)... La forme de raie de Wagner et al. est donnée par les équations (3.35), (3.36) de [57]. Lorsque $\gamma_{1}=\gamma_{2}$ dans ces équations, c'est-à-dire lorsque $\mathcal{H}_{0} / k_{\mathrm{B}} T \ll 1$ (cf. Eqs. (4.4) de [63]) elles se réduisent comme attendu aux équations (66)-(67) ci-dessus $\left(\gamma_{1}(\omega)=\gamma_{2}(\omega)=\right.$ $\operatorname{Re}(X+Y))$.

Cette expérience avait pour but d'observer la déviation du taux de relaxation électronique par rapport à la loi de Korringa au-dessous de la température de transition supraconductrice (cf. expérience de Hebel et Slichter sur le taux de relaxation nucléaire). Dans un supraconducteur les particularités de la densité d'états des quasi-particules devraient entraîner une divergence de la densité spectrale $I(\omega)$ et donc de $1 / T_{1 s}$, mais cette divergence est supprimée par l'anisotropie du gap. Il se trouve que l'intervalle hyperfin $5 A / 2 \hbar$ de ${ }^{160}$ Dy dans le thorium est comparable à l'anisotropie du gap, ce qui doit permettre (contrairement au cas de la R.M.N.) de vérifier la dépendance en fréquence du taux de relaxation $1 / T_{1 s}$ (Eq. (53)) 
ou de $(X+Y)$ (Eq. (67)). Dans les références [53], [58], [59], les auteurs utilisent une approche de type matrice densité, mais la forme de raie qu'ils obtiennent est très lourde ce qui rend la vérification assez compliquée, alors qu'elle serait très facile avec l'équation (48) ci-dessus, qui est équivalente à la leur (cf. [56]). Ultérieurement, comme nous l'avons dit plus haut, la méthode de la résolvante a fourni [63] à ces mêmes auteurs une forme de raie qui pour $A / k_{\mathrm{B}} T \ll 1$ se réduit à l'équation (66). Par contre, dans ce dernier article Wagner et al. ont attribué la suppression de la divergence de $(X+Y)$ à la présence du terme $i \Gamma / 2$ dans l'argument des densités spectrales $I$ au second membre de l'équation (67), ce qui est peut-être discutable car il n'y a pas de terme équivalent dans le cas de la R.M.N.

N.B. : Au dénominateur de l'équation (3.35) de [63] le premier terme devrait s'écrire $\bar{\omega}\left(\bar{\omega}+\frac{A}{2}+i \gamma\right)$.

7.3 FORME DE RAIE À TRÈS BASSE TEMPÉRATURE, EN PRÉSENCE DE RÉORIENTATION, POUR UNE TRANSITION $2^{+} 0^{+}$(Hartmann-Boutron, non publié). - Elle s'écrit $(p=\Gamma / 2-i \omega)$ :

$$
I(\omega) \propto \operatorname{Re}\left\{\frac{\left(p+\frac{i A}{\hbar}\right) P_{3 / 2}(1 / \Gamma)+\left(p-\frac{3}{2} \frac{i A}{\hbar}\right) P_{5 / 2}(1 / \Gamma)+\bar{\gamma}}{p^{2}-\frac{i A}{2 \hbar} p+\bar{\gamma} p+\frac{3 A^{2}}{2 \hbar^{2}}-i \frac{3 A}{5 \hbar}\left(\gamma_{1}-\gamma_{2}\right)}\right\},
$$

avec (cf. [63] Eqs. (3.29)-(3.36)) :

$$
\begin{aligned}
& \bar{\gamma}(\omega)=\frac{2}{5} \gamma_{1}(\omega)+\frac{3}{5} \gamma_{2}(\omega) \\
& \gamma_{1}(\omega)=\gamma\left(\omega+\frac{3 A}{2 \hbar}\right)+\gamma\left(-\omega+\frac{A}{\hbar}\right) \\
& \gamma_{2}(\omega)=\gamma\left(-\omega-\frac{3 A}{2 \hbar}\right)+\gamma\left(\omega-\frac{A}{\hbar}\right),
\end{aligned}
$$

où $\gamma(\omega)$ est relié à $I^{\prime}(\omega)$, équation (57), par :

$$
\gamma(\omega)=\operatorname{Re} I^{\prime}\left(\omega+i \frac{\Gamma}{2}\right) .
$$

Lorsque $\Gamma=0$ et $\omega=\frac{A}{\hbar}$ ou $-\frac{3}{2} \frac{A}{\hbar}, \gamma_{1}(\omega)$ se réduit à $u+v$ et $\gamma_{2}(\omega)$ à $u+w$, équation (59), et la forme de raie devient identique à l'équation (60) obtenue dans le chapitre précédent. Ceci vérifie une fois de plus le raccord entre les méthodes de type " matrice densité " et « résolvante ».

7.4 REMARQUE SUR LES OPÉRATEURS TENSORIELS IRRÉDUCTIBLES. - Les méthodes de calcul correspondantes sont puissantes, mais ne s'appliquent qu'à deux cas :

- symétrie sphérique, cf. les traitements exposés dans ce chapitre et le précédent,

- petits effets de relaxation en présence d'un grand champ appliqué ou hyperfin (cette situation se rencontre souvent dans les expériences de corrélation angulaires perturbées, cf. [2] Chap. VII, p. 333, mais guère en Mössbauer).

Par contre, en présence d'un champ intermédiaire $\left(g \mu_{\mathrm{B}} H_{0} S_{z} \sim A I . S\right)$, et d'effets de relaxation importants, les opérateurs tensoriels irréductibles perdent la majeure partie de leur intérêt : les dimensions des matrices à diagonaliser restent élevées.

\section{Problèmes propres à la relaxation électronique dipôle-dipôle $\left({ }^{12}\right)$.}

Cette relaxation n'est pas une relaxation spin-réseau. Elle soulève un certain nombre de problèmes que nous allons essayer d'exposer sur des cas simples.

\subsection{SYSTÈMES CONCENTRÉS.}

8.1.1. Relaxation dipôle-dipôle en champ magnétique élevé. Comparaison avec la R.P.E. - Considérons dans un cristal deux ions 1 et 2 dont les niveaux électroniques les plus bas sont des multiplicités de spin $\mathbf{S}$ de moment $g \mu_{\mathrm{B}} \mathbf{S}$, possédant un couplage hyperfin $\mathfrak{H}_{\mathrm{hf}}=A \mathbf{I} . \mathbf{S}$ (ou $A_{g} \mathbf{I}_{g} \mathbf{S}$ ). Entre les moments électroniques existe une interaction dipôle-dipôle (cf. Abragam [20] Chap. IV) :

$$
\begin{aligned}
\mathscr{H}_{\mathrm{dip}-\mathrm{dip}}=A_{d}+B_{d}+C_{d}+D_{d}+E_{d}+F_{d} \\
A_{d}=\frac{g^{2} \mu_{\mathrm{B}}^{2}}{r^{3}}\left(1-3 \cos ^{2} \theta\right) S_{1 z} S_{2 z}, \\
B_{d}=\frac{g^{2} \mu_{\mathrm{B}}^{2}}{r^{3}}\left\{-\frac{1}{4}\left(1-3 \cos ^{2} \theta\right) \times\right. \\
\left.\quad \times\left(S_{1+} S_{2-}+S_{1-} S_{2+}\right)\right\},
\end{aligned}
$$

$C_{d}=D_{d}^{\dagger}=\frac{g^{2} \mu_{\mathrm{B}}^{2}}{r^{3}}\left[-\frac{3}{2} \sin \theta \cos \theta \mathrm{e}^{-i \phi}\right] \times$

$$
\times\left[S_{1 z} S_{2+}+S_{1+} S_{2 z}\right],
$$

$E_{d}=F_{d}^{\dagger}=\frac{g^{2} \mu_{\mathrm{B}}^{2}}{r^{3}}\left[-\frac{3}{4} \sin ^{4} \theta \mathrm{e}^{-2 i \phi}\right]\left[S_{1+} S_{2+}\right]$,

$\left({ }^{12}\right)$ Basé sur un séminaire donné par l'auteur à Munich en juillet 1977. 
que nous allons supposer grande par rapport à $\mathscr{H}_{\mathrm{hf}}$. Imaginons de surcroît que les moments électroniques sont soumis à un grand champ extérieur :

$$
\mathscr{H}_{0}=-g \mu_{\mathrm{B}} H_{0}\left\{\begin{array}{l}
S_{1 z} \\
S_{2 z}
\end{array}\right\} \gg \mathscr{H}_{\mathrm{dip}} \gg A
$$

En présence de cet effet Zeeman électronique les niveaux hyperfins sont sensiblement les mêmes que si le hamiltonien hyperfin était réduit à sa partie diagonale :

$$
\mathcal{H}_{\mathrm{hf}}^{\prime}=A I_{\mathrm{z}} S_{\mathrm{z}} \quad\left(\text { ou } A_{g} I_{g \mathrm{z}} S_{\mathrm{z}}\right)
$$

et, en l'absence de relaxation, le spectre Mössbauer sera une superposition de spectres Zeeman correspondant à $S_{z}=-S, \ldots,+S$. En fait, l'interaction dipôledipôle va induire une relaxation entre les niveaux Zeeman électroniques. L'effet de cette relaxation sur le spectre Mössbauer de l'ion 1 peut se calculer aisément par la théorie stochastique d'Anderson si l'on connaît les probabilités de transition $W_{m_{1 m^{\prime} 1}}$ entre les niveaux Zeeman $\left(S_{z}=m_{1} ; m_{1}^{\prime}\right)$ de cet ion. En principe, tous les termes non diagonaux de $\mathscr{H}_{\text {dip }}, B_{d}, C_{d}$, $D_{d}, E_{d}, F_{d}$ contribuent à $W_{m_{1}, m^{\prime} 1}$, mais seul $B_{d}$ (terme " flip-flop ») conserve l'énergie Zeeman et donne une contribution importante.

La probabilité pour que, sous l'effet de $B_{d}$, l'ion 1 saute de $m_{1}$ à $m_{1}^{\prime}$ fixés, tandis que l'ion 2 saute de $m_{2}$ à $m_{2}^{\prime}$ quelconques, est donnée par la règle d'or de Fermi :

$$
W_{m_{1} \rightarrow m_{1}^{\prime}}=\frac{2 \pi}{\hbar} \rho(0) \sum_{\substack{m_{2} \\ m_{2}^{\prime}}} p_{m_{2}}\left|\left\langle m_{1}^{\prime} m_{2}^{\prime}\left|B_{d}\right| m_{1} m_{2}\right\rangle\right|^{2}
$$

où $p_{m_{2}}$ est la probabilité de trouver l'ion 2 dans l'état initial $m_{2}$ :

$$
p_{m_{2}}=\frac{1}{2 S+1} \quad \text { si } \quad k_{\mathrm{B}} T \gg g \mu_{\mathrm{B}} H_{0},
$$

(ce que nous supposerons ici) et $\rho(0)$ est la valeur à $E=0$ (conservation de l'énergie dans le processus $B_{d}$ ) de la densité d'états finals $\rho(E)$ associée à l'élargissement des niveaux $m_{1}, m_{1}^{\prime}, m_{2}, m_{2}^{\prime}$ par le champ dipolaire des autres ions du cristal. Il est en effet connu qu'on ne peut avoir de transition irréversible que s'il existe un continuum d'états finals : pour deux ions isolés, il n'y aurait pas de relaxation. En pratique, les autres ions contribuent à la fois à fournir un $\rho(0)$ et à faire sauter l'ion 1 de $m_{1}$ à $m_{1}^{\prime}$. Nous écrirons donc, en sommant sur les ions $i$ autres que 1 :

$$
W_{m_{1} \rightarrow m^{\prime} 1}=\frac{2 \pi}{\hbar} \rho(0) \sum_{\substack{i \\ m_{i}, m_{i}^{\prime}}} p_{m_{i}}\left|\left\langle m_{1}^{\prime} m_{i}^{\prime}\left|B_{d}\right| m_{1} m_{i}\right\rangle\right|^{2} .
$$

Il est généralement admis que la densité d'états $\rho(E)$ est bien représentée par une gaussienne

$$
\rho(E)=\frac{1}{\sqrt{2 \pi} \Delta E} \exp \left(-\frac{E^{2}}{\Delta E^{2}}\right)
$$

d'où

$$
\rho(0)=\frac{1}{\sqrt{2 \pi} \Delta E}\left(\simeq \frac{1}{\pi \Delta E}\right)
$$

Il reste à calculer l'élargissement $\Delta E$. Il provient du terme diagonal $A_{d}(1, i)$ de $\mathscr{H}_{\text {dip }}$, que nous pouvons mettre sous la forme :

$$
A_{d}(1, i)=-g \mu_{\mathrm{B}} S_{1 z} H_{z}(i),
$$

où le champ dipolaire $H_{z}(i)$ créé par l'ion $i$ au niveau de l'ion 1 dépend de la valeur propre $m_{i}$ de $S_{i z} ;\left\langle H_{z}(i)\right\rangle=0$ mais $\left\langle H_{z}(i)^{2}\right\rangle \neq 0$. Si nous définissons :

$$
\begin{aligned}
\bar{\delta}^{2} & =\sum_{i} \frac{g^{2} \mu_{\mathrm{B}}^{2}}{\hbar^{2}}\left\langle H_{z}(i)^{2}\right\rangle \\
& =\frac{1}{3} \frac{g^{4} \mu_{\mathrm{B}}^{4}}{\hbar^{2}} S(S+1) \sum_{j} \frac{\left(1-3 \cos ^{2} \theta_{1 i}\right)^{2}}{r_{1 i}^{6}} .
\end{aligned}
$$

$\Delta E$ est donné par :

$$
\Delta E=2 \hbar \sqrt{\delta^{2}}
$$

(le facteur 2 vient de ce qu'il faut ajouter les élargissements des niveaux des 2 ions).

Nous avons donc, avec $\rho(0) \simeq 1 / \pi \Delta E$

$$
\begin{aligned}
& W_{m_{1}-1 \rightarrow m_{1}}=\frac{2 \pi}{\pi\left(2 \sqrt{\delta^{2}}\right)} \sum_{i} \frac{g^{4} \mu_{\mathrm{B}}^{4}}{\hbar^{2}} \times \\
& \times\left[-\frac{1}{4} \frac{\left(1-3 \cos ^{2} \theta_{1 i}\right)^{2}}{r_{1 i}^{6}}\right] \\
& \times \sum_{m_{i}} p_{m_{i}+1}\left|\left\langle m_{1} m_{i}\left|S_{1}^{+} S_{i}^{-}\right| m_{1}-1, m_{i}+1\right\rangle\right|^{2}
\end{aligned}
$$

où $p_{m_{i}+1}=\frac{1}{2 S+1}$ si $m_{i}+1 \leqslant S$.

La somme sur $m_{i}$ est égale à

$$
\sum_{m_{i}}()=\frac{2 S(S+1)}{3}\left[S(S+1)-m_{1}\left(m_{1}-1\right)\right],
$$

et celle sur $i$ à

$$
\sum_{i}()=\frac{1}{8} \bar{\delta}^{2}\left[S(S+1)-m_{1}\left(m_{1}-1\right)\right],
$$

d'où finalement :

$$
W_{m_{1}-1 \rightarrow m_{1}}=\frac{1}{8} \sqrt{\overline{\delta^{2}}}\left[S(S+1)-m_{1}\left(m_{1}-1\right)\right] .
$$


Cette expression est le résultat d'un calcul de perturbations dans l'approximation du spectre blanc $\left(\sqrt{\bar{\delta}^{2}} \gg A\right)$. Pour que la méthode de perturbation soit valable, il faut que (cf. [1] Eq. (57))

$$
\frac{\left\langle\mathcal{H}_{1}^{2}\right\rangle}{\hbar^{2}} \tau_{\mathrm{c}}^{2} \ll 1 .
$$

Ici $\left\langle\mathcal{H}_{1}^{2}\right\rangle / \hbar^{2}$ est donné par la somme sur $i$, équation $(80)$, et :

$$
\tau_{\mathrm{c}}=\frac{\hbar}{\Delta E}=\frac{1}{2 \sqrt{\bar{\delta}^{2}}}
$$

donc

$$
\frac{\left\langle\mathcal{H}_{1}^{2}\right\rangle}{\hbar^{2}} \tau_{\mathrm{c}}^{2}=\frac{1}{32}\left[S(S+1)-m_{1}\left(m_{1}-1\right)\right],
$$

qui est bien petit par rapport à 1 lorsque $S$ n'est pas trop grand. Si les ions paramagnétiques occupent à $100 \%$ un réseau cubique simple dont le paramètre de maille est $d$ et, si l'échantillon est une poudre

$$
\overline{\delta^{2}}=\frac{g^{4} \mu_{\mathrm{B}}^{4}}{\hbar^{2}} \frac{S(S+1)}{3} \frac{6,8}{d^{6}}
$$

(cf. [20] p. 112, Eqs. (38) à (39')). Cette formule permet d'obtenir l'ordre de grandeur des probabilités $W_{m_{1} m_{1}^{\prime}}$ en fonction de $g, S$ et $d$. Si les ions paramagnétiques sont éloignés $(d \sim 10 \AA$ par exemple), $W$ sera petit par rapport à $A$ et le spectre restera résolu, mais avec des raies élargies. $\mathrm{Si}$, au contraire $d$ est petit, $W$ sera grand par rapport à $A$ et le spectre se réduira à une seule raie. Il ne faut cependant pas oublier que lorsque $d$ est de l'ordre de 2 à $3 \AA$, les spins sont aussi couplés par des interactions d'échange, généralement bien supérieures à $\mathcal{H}_{\mathrm{dip}}$ et à $g \mu_{\mathrm{B}} H_{0}$, si bien que les considérations précédentes ne sont plus valables (cf. Bonville et al. (1978) [48]).

Comparaison avec la résonance paramagnétique électronique. - Dans une expérience de R.P.E., on détecte une des composantes transverses du spin total de l'échantillon : $\mathbf{S}_{\mathrm{tot}}=\sum_{i} \mathbf{S}_{i}$. $\mathbf{S}_{\mathrm{tot}}$ commute avec $A_{d}+B_{d}$ et la relaxation spin-spin (caractérisant la variation du spin total) est due à $C_{d}+D_{d}+E_{d}+F_{d}$ qui ne conservent pas l'énergie. Le temps de relaxation correspondant [20bis] est $\left({ }^{13}\right)$ :

$$
\begin{aligned}
& \frac{1}{T_{12}} \sim \frac{2 \pi}{\hbar}\left\{\left\langle\left|C_{d}+D_{d}\right|^{2}\right\rangle \rho^{\prime}\left(\left|g \mu_{\mathrm{B}} H_{0}\right|\right)+\right. \\
&\left.+\left\langle\left|E_{d}+F_{d}\right|^{2}\right\rangle \rho^{\prime}\left(\left|2 g \mu_{\mathrm{B}} H_{0}\right|\right)\right\},
\end{aligned}
$$

$\left.{ }^{13}\right)$ Comme nous l'avons signalé plus haut, $W_{m_{1} m_{1}^{\prime}}$ contient aussi des contributions de ce genre: mais elles sont négligeables par rapport à celle de $B_{d}$. où, si nous supposons suivant la coutume que $\rho^{\prime}(E)$ est gaussienne :

$$
\rho^{\prime}\left(g \mu_{\mathbf{B}} H_{0}\right)=\frac{1}{\sqrt{2 \pi} \sqrt{\overline{4 \delta^{\prime 2}}}} \exp \left\{-\frac{\left(g \mu_{\mathbf{B}} H_{0}\right)^{2}}{\hbar^{2} \overline{4 \delta^{\prime 2}}}\right\} \text {. }
$$

Dans cette expression $\overline{\delta^{\prime 2}}$ est le second moment calculé en R.P.E. pour des spins identiques : $\overline{\delta^{2}}=\frac{9}{4} \overline{\delta^{2}}$. Compte tenu de notre hypothèse : $g \mu_{\mathrm{B}} H_{0} \gg \mathscr{H}_{\text {dip }} \sim$ $\sqrt{\overline{\delta^{2}}}, 1 / T_{12}$ va être très inférieur à $W_{m_{1} m_{1}^{\prime}}$.

Ces résultats montrent clairement que l'effet Mössbauer et la R.P.E. ne sont pas affectés de la même façon par la relaxation, contrairement à ce qui avait été initialement admis par quelques auteurs.

8.1.2 Relaxation dipôle-dipôle dans les composés ferriques en présence d'un grand hamiltonien de spin. Certains composés ferriques (biologiques ou analogues) possèdent un hamiltonien de spin inhabituellement grand

$$
H_{\mathrm{ss}}=D S_{z}^{2},
$$

avec $D>0$ de l'ordre de quelques $\mathrm{cm}^{-1}$ au lieu de $(1 / 100) \mathrm{cm}^{-1}$ usuellement. Cet hamiltonien donne naissance à 3 doublets électroniques dégénérés $| \pm 5 / 2\rangle,| \pm 3 / 2\rangle,| \pm 1 / 2\rangle$.

Il n'existe pas de processus de relaxation dipôledipôle à l'intérieur des doublets $| \pm 5 / 2\rangle$ et $| \pm 3 / 2\rangle$ car $S_{+}$et $S_{-}$y sont nuls. Par contre, l'ion peut sauter entre les trois doublets $| \pm 1 / 2\rangle,| \pm 3 / 2\rangle,| \pm 5 / 2\rangle$ sous l'effet de l'interaction flip-flop $B_{d}$ qui conserve l'énergie. Enfin, à l'intérieur du doublet dégénéré $| \pm 1 / 2\rangle$ la condition de conservation de l'énergie ne joue pas et les contributions à la relaxation de tous les termes non diagonaux de $\mathcal{H}_{\mathrm{dip}}: B_{d}, C_{d}, D_{d}, E_{d}$ et $F_{d}$ sont comparables.

Dans ces composés ferriques, le noyau ${ }^{57} \mathrm{Fe}$ est soumis à la fois à un gradient quadrupolaire statique dû au réseau et à un couplage hyperfin fluctuant $\mathcal{H}_{\mathrm{hf}}=A_{\|} I_{z} S_{z}(t)+A_{\perp} \mathbf{I}_{\perp} \cdot S_{\perp}(t)$.

Le premier calcul des spectres Mössbauer, dû à Blume [20ter], utilisait un hamiltonien hyperfin tronqué $\left(A_{1}=0\right)$ et ne prenait en compte que les processus $B_{d}$. Il ne comportait aucune discussion de $\rho(0)$ qui était incorporé à une constante de proportionnalité. On trouve alors que l'évolution thermique des spectres est due au peuplement progressif des niveaux électroniques excités (lequel intervient dans $W_{m_{1} m^{\prime}}$ par l'intermédiaire des $p_{m_{i}}$ ) lorsque $k_{\mathrm{B}} T$ devient comparable à $D$.

Ce traitement a été amélioré par Dattagupta (1979) [22] qui prend en compte l'interaction hyperfine complète et l'ensemble des processus dipolaires. Par contre, ses formules (Eq. (51) de [22]) ne sont valables que dans la limite rapide. D'autre part, la constante $C$ dans l'article de Dattagupta a une équation aux dimensions incorrecte car elle ne contient pas la den- 
sité d'états finals $\rho(0)$. Cette omission n'a cependant pas de conséquences graves sur ses résultats car il a ajusté, non pas $C$, mais l'un des $W_{m m^{\prime}}(\lambda$ dans l'Eq. (49) de [22]). Dattagupta trouve que le spectre Mössbauer est un doublet asymétrique, dont l'asymétrie s'inverse quand la température croît.

Signalons toutefois que si l'on introduit un $\rho(0)=1 /(\sqrt{2 \pi} \Delta E)$ avec $\Delta E \propto \sqrt{\left\langle H_{z}^{2}\right\rangle} \propto \sqrt{\left\langle B_{d}^{2}\right\rangle}$, ce $\Delta E$ va varier par un facteur $5 / \sqrt{3} \simeq 3$ entre $k_{\mathrm{B}} T=0$, où seul le doublet $| \pm 1 / 2\rangle$ est peuplé, et $k_{\mathrm{B}} T \gg D$ où les trois doublets sont équipeuplés, ce qui peut modifier assez sérieusement la température à laquelle se produit l'inversion d'asymétrie. Notons aussi que les fréquences $\lambda$ adoptées par Dattagupta correspondent à des temps caractéristiques anormalement courts pour des interactions dipôle-dipôle $\left(\lambda=20 \mathrm{~cm}^{-1}\right.$ équivaut à $\tau \sim 0,3 \times 10^{-12} \mathrm{~s}$ alors qu'on s'attendrait plutôt à $10^{-10} \mathrm{~s}$ ).

8.1.3 Relaxation dipôle-dipôle en symétrie cubique et champ nul. - Elle intervient dans l'étude Mössbauer (via ${ }^{170} \mathrm{Yb}$ ) de $\mathrm{Cs}_{2} \mathrm{NaYbCl}_{6}$.

C'est un cas difficile car il n'y a pas de direction physique à laquelle on puisse se référer pour décomposer $\mathscr{H}_{\text {dip }}$ en termes diagonaux et non diagonaux. Pour tourner la difficulté, Shenoy et al. [36], [41] ont utilisé un modèle phénoménologique dans lequel l'effet, sur un spin électronique, de son couplage dipolaire à tous les autres spins est représenté par un champ fluctuant isotrope aléatoire possédant une fonction de corrélation exponentielle (cf. Eq. (62) ci-dessus) :

$$
\left\langle H_{1}(0) H_{1}(t)\right\rangle=\left\langle H_{1}^{2}\right\rangle \mathrm{e}^{-t / \tau_{c}}
$$

où $\hbar / \tau_{\mathrm{c}}$ est l'équivalent de notre $\Delta E$ antérieur. Ce modèle a été appliqué aux spectres de $\mathrm{Cs}_{2} \mathrm{NaYbCl}_{6}$ où, bien que le composé soit concentré, l'approximation du spectre blanc n'est pas valable car les ions magnétiques sont éloignés $(10 \AA)$ ) si bien que $1 / \tau_{\mathrm{c}} \sim A / \hbar$. Cependant le fait qu'en présence d'un petit champ magnétique $H_{0}$, les paramètres $\left\langle H_{1}^{2}\right\rangle$ et $\tau_{\mathrm{c}}$ déduits des spectres paraissent dépendre de $H_{0}$, indique le caractère approximatif du modèle. En réalité, les fonctions de corrélation dipolaires sont non exponentielles et anisotropes $\left({ }^{14}\right)$. Inversement, cf. $\S 7.1$, Afanasev et al. [44], [45] ont tiré $I(\omega)$ des résultats expérimentaux par inversion des spectres; ils trouvent que $I(\omega)$ est proche d'une gaussienne.

8.2 SystèMES DILUÉs. - Lorsque les moments électroniques n'occupent qu'une fraction $c$ des sites, les sommations sur $i$ dans $W_{m_{1} m^{\prime}}$, équation (80), et dans $\overline{\delta^{2}}$, equation (79), sont multipliées par $c$ et $W_{m_{1} m_{1}^{\prime}}$ est donc multiplié par $\sqrt{c}:$ les effets de relaxation sont réduits par la dilution. En ce qui concerne les calculs, il convient de ne pas oublier que si $\overline{\delta^{2}}$ diminue, l'approximation du spectre blanc $\left(\sqrt{\delta^{2}} \gg A\right)$ risque

$\left({ }^{14}\right)$ Voir la référence [15] de [36], la discussion p. 588 et les références [16-22] de [45]. de ne plus être satisfaite. Par contre, $\left\langle\mathscr{H}_{1}^{2}\right\rangle \propto c$ et $\tau_{\mathrm{c}}^{2} \propto 1 / \overline{\delta^{2}} \propto 1 / c$, si bien que la validité du traitement de perturbation n'est pas affectée par la dilution.

\section{Conclusion.}

Les théories que nous venons d'exposer sont relativement compliquées et comportent une lourde algèbre. Il s'agit cependant là d'inconvénients mineurs en regard des avantages qu'offre l'effet Mössbauer comme moyen d'étude de la relaxation :

- l'atome Mössbauer est une sonde locale, alors que par exemple la R.P.E. observe le mode uniforme des spins électroniques, qui n'est pas nécessairement affecté de la même façon par la relaxation (cas de la relaxation dipôle-dipôle électronique);

- l'effet Mössbauer est une méthode spectroscopique et, en présence de spectres élargis, il est souvent possible de distinguer entre élargissements statique (inhomogène) et dynamique (relaxation), en se basant sur les différences d'aspect et d'évolution thermique des spectres correspondant à ces deux types de processus. Cette remarque est importante pour l'étude des verres de spin;

- l'effet Mössbauer fournit deux fenêtres en temps :

- $\tau \sim \tau_{\mathrm{hf}} \quad$ (période hyperfine) $\sim 10^{-8}-10^{-10} \mathrm{~s}$ et (dans des cas particuliers) :

- $\tau \sim \tau_{\mathrm{n}}$ (durée de vie nucléaire) $\sim 10^{-7}-10^{-9} \mathrm{~s}$ qui s'intercalent entre celles des muons $\left(\sim 10^{-6} \mathrm{~s}\right)$ et des neutrons $\left(\sim 10^{-10}-10^{-12} \mathrm{~s}\right)$;

- l'effet Mössbauer est très bien adapté à l'étude des phénomènes d'activation, de diffusion, etc...

- En ce qui concerne la relaxation électronique (cf. YbAu), l'effet Mössbauer fournit directement $1 / T_{1 s}$ alors que la largeur de raie R.P.E. est de la forme $\gamma \Delta H=C+1 / T_{1 s}$ (où la constante $C$ dépend de la concentration et de la fréquence) ce qui rend difficile l'observation de déviations Kondo.

- Dans les supraconducteurs, l'effet Mössbauer permet d'opérer en l'absence de champ. Par ailleurs (cf. ThDy) la constante de structure hyperfine $A$ est comparable à l'anisotropie du gap. Il a ainsi été possible de vérifier la dépendance en fréquence du temps de relaxation électronique $T_{1 s}$, chose qui n'avait pu être faite sur le temps de relaxation nucléaire, car la fréquence de Larmor nucléaire est trop basse.

- Il faut, enfin, mentionner les belles expériences sur les sources Mössbauer à basse température, qui permettent de travailler à très faible concentration et hors équilibre thermique. Il a ainsi été possible d'étudier de façon approfondie tant l'effet JahnTeller de $\mathrm{Fe}^{++}$dans $\mathrm{ZnS}$, que l'effet Kondo de $\mathrm{Yb}^{3+}$ dans l'or. Ces expériences et d'autres, également intéressantes, sont décrites en détail dans la revue d'Imbert ci-après. 


\section{Bibliographie}

Cette bibliographie est divisée en deux :

- d'une part des références anciennes non classées [4-20], extraites d'une bibliographie plus complète qui se trouve dans la référence [2] p. 329 ,

- d'autre part, des références récentes (depuis 1979) classées par ordre chronologique $[21 \ldots]$ et commentées.

[1] Hartmann-Boutron, F., Article précédent.

[2] Hartmann-Boutron, F., Ann. Phys. 9 (1975) 285, voir p. 310.

Corrections à cet article :

p. 314. Nous avons dit que l'on ne voyait jamais de structure hyperfine magnétique dans les composés ferreux, en dehors des phases magnétiques ordonnées : en fait, des spectres de relaxation lente ont pu être observés dans des cas particuliers, même en champ nul. Voir :

- ZimmermanN, R., Ritter, G., Spiering, H., Nagy, D. L., J. Physique Colloq. 35 (1974) C6-439 (Conf. Möss. Bendor).

- Abeledo, C. R., Frankel, R. B., Misetich, A. A., Chem. Phys. Lett. 31 (1975) 108.

- Price, D. C., Srivastava, K. K. P., J. Physique Colloq. 37 (1976) C6-123 (Conf. Möss. Corfou)

etc..

p. 319 : La théorie stochastique de Dattagupta et Blume est examinée dans le $\S$ VII-4D, p. 338. Le texte de la page 339 est mal disposé : en haut de la 2 e colonne, à la place de exemple 2, il faudrait lire :

« F | Extension du modèle de Dattagupta et Blume au cas Mössbauer. Ce modèle conduit à une relation identique à VII-100, mais avec un $G_{L}^{0}$ différent ([24] Eq. (39)). Il s'applique, entre autres, aux fluctuations du gradient quadrupolaire dans les liquides et permet d'interpréter les spectres Mössbauer au voisinage de la transition verre $\rightarrow$ liquide visqueux (cf. [24] Eq. (44)).»
[3] Dattagupta, S., Hyp. Int. 11 (1981) 77-126.

[4] Blume, M. dans Hyperfine Structure and Nuclear Radiation (Eds. Matthias Shirley, North Holland) 1968, p. 911.

[5] Hartmann-Boutron, F., SpanjaArd, D., J. Physique 33 (1972) 285.

[6] GonZalez-Jimenez, F., Imbert, P., HartmanNBoutron, F., Phys. Rev. B 9 (1974) 95. Erratum : Phys. Rev. B 10, 2134 (1974).

[7] Hartmann-Boutron, F., Phys. Rev. B 10 (1974) 2113.

[8] GonZalez-Jimenez, F., HaRTMann-Boutron, F., IMBert, P., Phys. Rev. B 10 (1974) 2122.

[9] Clauser, M. J., Blume, M., Phys. Rev. B 3 (1971) 583.

[10] Blume, M., Tuon, J. A., Phys. Rev. 165 (1968) 446.

[11] Blume, M., Phys. Rev. 174 (1968) 351.

[12] Dattagupta, S., Blume, M., Phys. Rev. B 10 (1974) 4540.

[13] Scherer, C., Nucl. Phys. A 157 (1970) 81.

La distribution de Poisson adoptée par Scherer est incorrecte. Cette erreur a été corrigée par Blume dans la référence [14].

[14] Blume, M., Nucl. Phys. A 167 (1971) 81.

[15] Hirst, L. L., J. Phys. Chem. Sol. 31 (1970) 655.

[16] Fano, U., Phys. Rev. 131 (1963) 259.

[17] Gabriel, H., Bosse, J., Rander, K., Phys. Stat. Sol. 27 (1968) 301.

[18] Afanasev, A. M., Gorobchenko, V. D., Soviet Phys. J.E.T.P. 39 (1974) 690.

[19] Bradford, E., Marshall, W., Proc. Phys. Soc. 87 (1966) 831.

[20] Abragam, A., The Principles of Nuclear Magnetism (Oxford University Press) 1961.

[20bis] CASPERS, W. J., Theory of spin relaxation (Interscience NY) 1964.

[20ter] Blume, M., Phys. Rev. Lett. 18 (1967) 305.

\section{Bibliographie récente}

(Classée par année : les articles examinés en détail dans le texte portent deux astérisques, les articles mentionnés, un seul.)

1975

[21] Dattagupta, S., Phys. Rev. B 12 (1975) 47.

Effect of Nuclear Motion on Mössbauer Spectra.

Effect d'un mouvement spatial non borné du noyau, s'accompagnant d'une modification de l'environnement (par exemple dans un liquide). Méthode de Clauser Blume. Résultat général : Eq. (28). Approximation R.P.A. (collisions fortes) : Eq. (36). Hypothèse isotrope Eqs. (45)-(49).

*[22] Dattagupta, S., Phys. Rev. B 12 (1975) 3584.

Effect of off-diagonal hyperfine interaction on Mössbauer relaxation spectra.

*[23] Hartmann-Boutron F., Spanjaard, D., J. Physique 36 (1975) 307.

On the use of Liouville relaxation supermatrices in Mössbauer studies.
L'appendice I de cet article indique la règle générale permettant de passer des $U_{0}$ et $U_{0}^{+}$aux $\mathcal{U}_{0}$ puis aux $\bar{V}$ lorsque la relaxation intervient plus d'une fois dans le problème (cas de la source à basse température et de la diffusion, cf. Réf. [33] II ci-dessous).

*[24] Sivardière, J., Blume, M., Clauser, M. J., Hyp. Int. 1 (1975) 227.

Magnetic relaxation and paramagnetic Mössbauer spectra : influence of the off-diagonal hyperfine coupling.

*25] Sivardière, J., Blume, M., Hyp. Int. 1 (1975) 283.

Paramagnetic Mössbauer spectra of ${ }^{161} \mathrm{Dy}\left(\Gamma_{6}\right.$ or $\left.\Gamma_{7}\right)$ and ${ }^{166} \mathrm{Er}\left(\Gamma_{8}\right)$ in cubic symmetry : influence of relaxation. 
1976

[26a]Afanas'ev, A. M., Onishenko, E. V., Soviet Phys. JETP 43 (1976) 222.

Relaxation Mössbauer spectra in cubic systems.

Relaxation de particules superparamagnétiques cubiques : champ hyperfin sautant entre les axes quaternaires (Eqs. (10)-(11)) ou ternaires(Eqs. (10)(9)-(12)). Ce traitement est réexaminé dans la thèse d'Etat de P. Bonville (1981) [60] : l'affirmation suivant laquelle, "en symétrie cubique, le spectre d'absorption ne dépend pas du vecteur de polarisation $\eta$ du rayonnement incident, si bien qu'on peut choisir l'axe de quantification suivant $\eta "$, ne paraît pas correcte - comme le montre d'ailleurs l'équation (15) de [45]. Cette affirmation n'est valable que pour une symétrie sphérique.

[26b] Manykin, E. A., Onishenko, E. V., Soviet Phys. Solid State 18 (1976) 1870.

Mössbauer relaxation spectra of superparamagnetic particles.

Extension de [26a] au cas où il existe un gradient quadrupolaire non nul.

[27] Afanas'ev, A. M., Gorobchenko, V. D., Phys. Status Solidi (b) 73 (1976) 73.

On the theory of Mössbauer emission relaxation spectra.

Nouvelle dérivation de la forme de raie d'une source à basse température (cf. [4], [5], [7]), avec la méthode de la résolvante. Résultat général, Eq. (10), peut-être contestable, cf. [50], p. 61 .

[28] Afanas'ev, A. M., Gorobchenko, V. D., Phys. Status Solidi b 76 (1976) 465.

Theory of time dependent Mössbauer emission spectra under relaxation conditions.

[29] Blume, M., J. Physique Colloq. 37 (1976) C6-61 (Conf. Mössbauer Corfou).

Recent results in relaxation theory : non stationary processes and polarization effects.

*[30] Chopin, C., SpanjaARd, D., HaRtmann-Boutron, F., J. Physique Colloq. 37 (1976) C6-73 (Corfou).

Relaxation supermatrices and relaxation models in the interpretation of Mössbauer paramagnetic relaxation spectra.

Suite de [23].

[31] Dattagupta, S., Phys. Rev. B 14 (1976) 1329.

Effect of molecular motions in liquids on Mössbauer relaxation spectra.

Suite de [21]. Les mouvements de translation et rotation de la molécule dans le liquide sont supposés décrits par des équations de diffusion. Forme de raie : Eqs. (17)-(22). Expression analytique : Eq. (25).

[32] Dattagupta, S., Philos Mag. 33 (1976) 59.

Study by Mössbauer effect of diffusion of interstitials (C or N) in Austenite - A theoretical treatment.

Application de la théorie de Blume (1968). Calcul de la forme de raie lorsque l'atome ${ }^{57} \mathrm{Fe}$ peut avoir (ou non) un premier voisin intersticiel qui saute entre six positions équivalentes. Forme de raie analytique : Eq. (38).

*[33] Hartmann-Boutron, F., J. Physique 37 (1976) 533, 549.

Relaxation effects in Mössbauer transmission and scattering I General formulas II Introduction of relaxation.
Méthode "de la matrice densité ». Les formules de l'article II relatives à la diffusion, qui sont très lourdes mais très explicites, ont été jugées par certains auteurs "plus faciles à mettre sur ordinateur " que les expressions formelles compactes obtenues antérieurement par Afanasév et Gorobchenko (1974) avec la méthode de la résolvante (la façon d'écrire les éléments de matrice de telles expressions n'est en effet pas toujours évidente).

Erratum : au second membre de l'équation (64) de 33.I, dans le premier terme, il faut rajouter $\sigma_{\mathbf{g}_{18_{1}}}$ après $\sum_{\mathbf{B}_{184}}$

[34] Kankelett, E., Kording, A., J. Physique colloq. 37 (1976) C6-65 (Corfou).

The two photon two intermediate state decay, or the effect of coherent relaxation on Mössbauer spectra.

[35] Meyer, C., Hartmann-Boutron, F., SpanjaArd, D., J. Physique colloq. 37 (1976) C6-61 (Corfou).

Mössbauer spectra in the presence of electronic relaxation in a quadruplet $\Gamma_{8}$ of rare earth.

La relaxation est supposée due au couplage $-2\left(g_{\mathrm{J}}-1\right) \boldsymbol{g}_{\mathrm{sf}} \mathbf{J} . \mathbf{s}$ de $\mathbf{J}$ aux électrons de conduction, alors que [25] utilise un modèle stochastique peut-être moins réaliste. Le spectre est obtenu par inversion d'une matrice $80 \times 80$.

*[36] Shenoy, G. K., Dunlap, B. D., Dattagupta, S., AsCh, L., Phys. Rev. Lett. 37 (1976) 539.

Calculation of Mössbauer paramagnetic relaxation spectra without the "White Noise Approximation ".

[37] Winkler, H., Heinrich, H. J., Gerdau, E., J. Physique colloq. 37 (1976) C6-261 (Corfou).

Relaxation phenomena in ferrofluids.

Traitement analogue à celui de Dattagupta et Blume (cf. H. Winkler, Zeits. f. Physik A 276 (1976) 225).

1977

[38] Banerjee, S., Blume, M., Phys. Rev. B 16 (1977) 3061.

Polarization analysis of emission lines in the presence of relaxation.

[39] Chugunova, G. P., Mitin, A. V., Phys. Status Solidi $b 81$ (1977) 69.

Dynamic theory of Conversion Electron Mössbauer Spectroscopy.

*[40] Dattagupta, S., Phys. Rev. B 16 (1977) 158.

Stochastic model for classical bath variables and its influence on line shape expression.

Modèle stochastique sans WNA.

*[41] Dattagupta, S., Shenoy, G. K., Dunlap, B. D., AsCh, L., Phys. Rev. B 16 (1977) 3893.

Breakdown of the "White Noise Approximation" in the Mössbauer relaxation spectra : the case of $\mathrm{Cs}_{2} \mathrm{NaYbCl}_{6}$.

Méthode de la résolvante. Théorie correspondant à la réf. [36] ci-dessus.

[42] Dattagupta, S., Solid State Commun. 24 (1977) 19.

Self interstitials trapped at Co impurities in electron irradiated $\mathrm{Al}$ : theoretical study of the effects of static and dynamic quadrupolar interactions on Mössbauer line.

Traitement de type Blume et Tjon (1968). Voir aussi [68]. 
[43] Hartmann-Boutron, F., Spanjaard, D., J. Physique 38 (1977) 961.

Interference effects in Mössbauer relaxation spectra.

Calcul des petites asymétries de forme de raie dues à l'interférence entre l'effet photoélectrique et la conversion interne.

1978

*[44] Afanas'ev, A. M., Onishenko, E. V., Asch, L., Kalvius, G. M., Phys. Rev. Lett. 40 (1978) 816.

Frequency dependence of the spin-spin relaxation rate from Mössbauer spectroscopy.

Relaxation dipôle-dipôle dans $\mathrm{Cs}_{2} \mathrm{NaYbCl}_{6}$.

*[45] Afanas'ev, A. M., Onishenko, E. V., Soviet Phys. JETP 47 (1978) 585.

Theory of Mössbauer spectra in the presence of spinspin relaxation.

Théorie correspondant à l'article précédent [44].

*[46] Afanas'ev, A. M., Litterst, F. J., Gierisch, W., J. Physique Lett. 39 (1978) 183.

Determination of the ${ }^{57} \mathrm{Co}$ hyperfine field in $\mathrm{Pd}\left({ }^{57} \mathrm{Co}\right)$ from Mössbauer emission spectra in the regime of fast relaxation.

Cascade ${ }^{57} \mathrm{Co} \rightarrow{ }^{57} \mathrm{Fe}$ avec réorientation dans les états intermédiaires.

[47] Blume, M., J. Math. Phys. 19 (1978) 2004.

Comment on quantum systems subject to random pulses.

*[48] Bonville, P., Hodges, J. A., Imbert, P., HartmanNBoutron, F., Phys. Rev. B 18 (1978) 2196.

Spin-spin and spin lattice relaxation of $\mathrm{Yb}^{3+}$ in $\mathrm{YbAlO}_{3}, \mathrm{TmAlO}_{3}: \mathrm{Yb}$ and $\mathrm{YbAlO}_{3}: \mathrm{Yb}$ and magnetic ordering in $\mathrm{YbAlO}_{3}$ measured by the Mössbauer effect.

[49] Dattagupta, S., Hyp. Int. 4 (1978) 942 (Madison). Hyperfine spectra in the presence of relaxation.

\section{9}

[50] Banerjee, S., J. Appl. Phys. 50 (1979) 7581.

The Clauser Blume model for the Mössbauer lineshape with non stationary relaxation effects.

*[51] Hartmann-Boutron, F., J. Physique 40 (1979) 57.

On the use of Liouville relaxation supermatrices in Mössbauer studies. II.

Suite de [23] [30].

*[52] Litterst, F. J., Afanasev, A. M., Gorobchenko, V. D., Kalvius, G. M., J. Phys. C 12 (1979) 5551.

Phenomenological description of the relaxation effects observed by Mössbauer emission spectroscopy on very dilute $\mathrm{Pd}\left({ }^{57} \mathrm{Co}\right)$.

Suite de [46].

*53] Wagner, W., Dissertation. Technische Universität München (1979).

Mössbauer Untersuchungen an verdünnten paramagnetischen $\mathrm{Dy}^{3+}$ Ionen in normal-und supraleitenden Metallen.

Forme de raie de type « matrice densité » : voir p. 40.
1980

[54] Afanas'ev, A. M., Gorobchenko, V. D., Peregudov, V. N., Soviet Phys. Solid State 22 (1980) 1315.

Mössbauer emission spectra for decays with a change in the electric field gradient in the relaxation process.

*[55] Hartmann-Boutron, F., J. Phys. Colloq. 41 (1980) C1-223 (Conférence Mössbauer Portoroz).

Some new results in Mössbauer relaxation theory.

Forme de raie de $\mathrm{Yb}^{170}$ en symétrie uniaxiale quand la W.N.A. n'est pas valable : Eq. (3). Largeur de raie dans la limite rapide en présence d'un spin électronique fluctuant ou d'un gradient quadrupolaire fluctuant : Eqs. (4) (5).

*[56] Hartmann-Boutron, F., J. Phys. 41 (1980) 1289.

On the use of Liouville relaxation supermatrices in Mössbauer studies. III. Application to Mössbauer relaxation in superconductors.

Suite de [23], [30], [51].

*[57] MoruP, S., Dissertation Lyngby 1980.

Paramagnetic and superparamagnetic relaxation phenomena studied by Mössbauer spectroscopy.

*[58] Wagner, W., Kalvius, G. M., Gorobchenko, V. D., J. Magn. Magn. Mat. 15-18 (1980) 626 (ICM Munich).

Relaxation of paramagnetic Dy moments in type I superconductors.

*[59] Wagner, W., Kalvius, G. M., Gorobchenko, V. D., J. Phys. Colloq. 41 (1980) C1-243 (Portoroz).

Mössbauer study of the frequency dependence of paramagnetic relaxation of Dy moments in type I superconductor thorium.

1981

*[60] Bonville, P., Thèse de Doctorat d'Etat, Orsay 1981. Etude par effet Mössbauer des états de valence de l'ytterbium dans des composés métalliques et de la relaxation du moment paramagnétique de $\mathrm{Yb}^{3+}$ à très basse température.

*[61] Bonville, P., Garcin, C., Gerard, A., Imbert, P., Jehanno, G., Phys. Rev. B 23 (1981) 4293.

Mössbauer absorption and emission study of dilute $\mathrm{Fe}^{++}$impurities in cubic $\mathrm{ZnS}$. Observation of metastable electronic levels. A : Relaxation measurements.

*[62] DattaguPta, S., Hyp. Int. 11 (1981) 77-126.

Study of time-dependent hyperfine interactions by PAC, Mössbauer Effect, $\mu$ SR and NMR : a review of stochastic models.

Au second membre des équations (3.9), (6.1) de cet article, il faudrait lire $\left(\mathcal{U}^{0}\right)^{-1}$ au lieu de $\mathcal{U}^{0}$. Dans l'équation (4.43) il faudrait lire $\exp \left[-\frac{1}{3} h^{2} L(L+1) t\right]$. Les équations (5.2) et (5.3) devraient être numérotées (5.3), (5.4).

*[63] WAGner, W., Litterst, F. J., Gorobchenko, V. D., Afanas'ev, A. M., Kalvius, G. M., J. Physics F 11 (1981) 1959.

Enhancement of the electronic relaxation rate of the electronic spin of $\mathrm{Dy}^{3+}$ impurities in superconducting thorium studied by Mössbauer spectroscopy.

Suite de [53] [58] [59]. Forme de raie de type "résolvante $"$. 
$\underline{1982}$

[64] Afanas'ev, A. M., Yakovleva, O. A., Hyp. Int. 12 (1982) 105.

Mössbauer Spectra of the $\Gamma_{8}$ quadruplet in the presence of spin lattice relaxation.

Cet article montre que pour une forme particulière du hamiltonien de relaxation :

$\mathscr{H}_{1}=d_{x x}(t) S_{x}^{2}+d_{y y}(t) S_{y}^{2}+d_{z z}(t)-S_{z}^{2}$

(Eq. (14)), la structure hyperfine de $\Gamma_{8}$ ne disparaît pas entièrement dans la limite rapide, mais se réduit à celle d'un $\Gamma_{6}$ (deux fois). Cette nondestruction tient à ce que $\mathcal{J}_{1}$ commute avec une partie du hamiltonien hyperfin; les matrices $4 \times 4$ correspondant à cette partie invariante se factorisent en matrices $2 \times 2$ proportionnelles à celles d'un $\Gamma_{6}$.

[65] Afanas'ev, A. M., Yakovleva, O. A., Sedov, V. E., Hyp. Int. 12 (1982) 211.

Mössbauer relaxation spectra of the spherical super- paramagnetic particles with zero energy of anisotropy.

*[66] Asch, L., Potzel, W., Kalvius, G. M., Afanas'ev, A. M., Onishchenko, E. V., J. Magn. Magn. Mat. 29 (1982) 317.

Asymmetric frequency dependence of the spin-spin relaxation function in paramagnetic $\mathrm{Cs}_{2} \mathrm{NaYbCl}_{6}$.

[67] Wegener, H., Wimmer, K., SeYboth, D., Zeman, N., Hyp. Int. 12 (1982) 15.

A microscopic model for the spin-spin relaxation of $\mathrm{Fe}^{3+}$ in an amorphous sample.

\section{$\underline{1983}$}

[68] Litterst, F. J., Gorobchenko, V. D., Kalvius, G. M., Hyp. Int. 14 (1983) 21. Analytical solution for the ${ }^{57} \mathrm{Fe}$ Mössbauer spectra for octahedral cage hopping and correlated electric field gradient fluctuations. 